\title{
Determination of the small RNA GcvB regulon in the Gram-negative bacterial pathogen Pasteurella multocida and identification of the GcvB seed binding region
}

\author{
EMILY L. GULLIVER, ${ }^{1}$ AMY WRIGHT, ${ }^{1}$ DEANNA DEVESON LUCAS, ${ }^{1}$ MARIANNE MÉGROZ, ${ }^{1}$ ODED KLEIFELD, ${ }^{2}$ \\ RALF B. SCHITTENHELM, ${ }^{2}$ DAVID R. POWELL, ${ }^{3}$ TORSTEN SEEMANN, ${ }^{1,4}$ JÜRGEN B. BULITTA, ${ }^{5}$ \\ MARINA HARPER, ${ }^{1,6}$ and JOHN D. BOYCE ${ }^{1,6}$ \\ ${ }^{1}$ Infection and Immunity Program, Monash Biomedicine Discovery Institute and Department of Microbiology, Monash University, Clayton, \\ Victoria 3800, Australia \\ ${ }^{2}$ Monash Biomedical Proteomics Facility, Monash Biomedicine Discovery Institute and Department of Biochemistry and Molecular Biology, \\ Monash University, Clayton, Victoria 3800, Australia \\ ${ }^{3}$ Monash Bioinformatics Platform, Monash University, Clayton, Victoria 3800, Australia \\ ${ }^{4}$ Victorian Life Sciences Computation Initiative, The University of Melbourne, Parkville, Victoria 3052, Australia \\ ${ }^{5}$ Department of Pharmaceutics, College of Pharmacy, University of Florida, Orlando, Florida 32827, USA
}

\begin{abstract}
Pasteurella multocida is a Gram-negative bacterium responsible for many important animal diseases. While a number of $P$. multocida virulence factors have been identified, very little is known about how gene expression and protein production is regulated in this organism. Small RNA (sRNA) molecules are critical regulators that act by binding to specific mRNA targets, often in association with the RNA chaperone protein $\mathrm{Hfq}$. In this study, transcriptomic analysis of the $P$. multocida strain VP161 revealed a putative sRNA with high identity to GcvB from Escherichia coli and Salmonella enterica serovar Typhimurium. High-throughput quantitative liquid proteomics was used to compare the proteomes of the P. multocida VP161 wild-type strain, a $\operatorname{gcv} B$ mutant, and a GcvB overexpression strain. These analyses identified 46 proteins that displayed significant differential production after inactivation of $\operatorname{gcv} B, 36$ of which showed increased production. Of the 36 proteins that were repressed by GcvB, 27 were predicted to be involved in amino acid biosynthesis or transport. Bioinformatic analyses of putative $P$. multocida GcvB target mRNAs identified a strongly conserved 10 nucleotide consensus sequence, $5^{\prime}$-AACACAACAT-3' ${ }^{\prime}$, with the central eight nucleotides identical to the seed binding region present within GcvB mRNA targets in $E$. coli and $S$. Typhimurium. Using a defined set of seed region mutants, together with a two-plasmid reporter system that allowed for quantification of sRNA-mRNA interactions, this sequence was confirmed to be critical for the binding of the $P$. multocida GcvB to the target mRNA, gltA.
\end{abstract}

Keywords: GcvB; sRNA; Pasteurella multocida; Hfq; GltA

\section{INTRODUCTION}

Pasteurella multocida is a Gram-negative, coccobacillus that is the causative agent of many economically important diseases, including fowl cholera, swine atrophic rhinitis, hemorrhagic septicemia, and various respiratory diseases of ungulates (Wilkie et al. 2012). P. multocida produces several virulence factors that are critical for the bacterium to cause disease. These include primary virulence factors, such as the polysaccharide capsule, lipopolysaccharide (LPS), and filamentous hemagglutinin as well as virulence-associated factors, such as proteins involved in iron and nutrient acquisition (Fuller

${ }^{6}$ These authors contributed equally to this work. Corresponding author: john.boyce@monash.edu 117. et al. 2000; Bosch et al. 2002; Harper et al. 2004; Boyce and Adler 2006). Appropriate regulation of these factors is likely critical for $P$. multocida survival. For example, during P. multocida in vivo growth, the bacteria must acquire and/ or synthesize all necessary amino acids, many of which are not freely available in sufficient quantities (Boyce and Adler 2006). This requires the production of amino acid biosynthesis and transport proteins, the expression of which must be tightly regulated to ensure that there is a balance between energy input and expenditure.

(C) 2018 Gulliver et al. This article is distributed exclusively by the RNA Society for the first 12 months after the full-issue publication date (see http://rnajournal.cshlp.org/site/misc/terms.xhtml). After 12 months, it is available under a Creative Commons License (Attribution-NonCommercial 4.0 International), as described at http://creativecommons.org/licenses/by$\mathrm{nc} / 4.0 /$. 
Recently, we showed that the Hfq protein was essential for the appropriate expression of a range of proteins in the P. multocida serogroup A strain VP161, including those required for the biosynthesis of hyaluronic acid capsule which is a primary virulence factor (Mégroz et al. 2016). The Hfq protein is an RNA chaperone that directly interacts with particular small regulatory RNA (sRNA) molecules to facilitate their binding to specific mRNA targets. Noncoding sRNA molecules are generally 40-400 nucleotides (nt) long and regulate transcript/protein expression within bacteria by binding to target mRNA via complementary base pairing (Desnoyers et al. 2013). There is redundancy within the sRNA regulatory network, as one sRNA species may bind to many different mRNA targets and each mRNA target may be regulated by several sRNA species (Desnoyers et al. 2013). Depending on the type of interaction, the binding of a sRNA to a target mRNA may result in either inhibition or induction of protein production. The binding of the sRNA to the ribosome-binding site (RBS) of an mRNA target can block translation and therefore reduce protein production. Alternatively, sRNA binding can result in rapid mRNA degradation via induction of Ribonuclease E activity against double-stranded RNA (Gottesman and Storz 2011). Less commonly, protein production can be enhanced via the binding of the sRNA to a natural secondary structure region in the mRNA that normally acts to occlude the RBS. This sRNA-mRNA interaction leads to the unfolding of the secondary structure, allowing the ribosome greater access to the RBS in order to initiate translation (Gottesman and Storz 2011).

Comparative global transcriptomic and proteomic analyses of the P. multocida strain VP161 and an isogenic $h f q$ mutant revealed that many genes displayed altered transcript expression, and/or altered protein production, when $h f q$ was inactivated (Mégroz et al. 2016). Analysis of the transcriptional data also allowed for the identification of a number of intergenic regions encoding putative sRNAs (M Mégroz, unpubl.). One putative sRNA identified in strain VP161, which is also encoded on the Pm70 genome (GenBank AE004439.1, position 652175 to 651999), exhibited high sequence identity to the Hfq-dependent sRNA GcvB. In E. coli and Salmonella enterica serovar Typhimurium (S. Typhimurium) GcvB has been shown to negatively regulate the production of proteins involved in amino acid transport and biosynthesis, such as the amino acid transporters ArgT, BrnQ, DppA, OppA, SstT, TppB, and YaeC and the amino acid biosynthesis proteins GdhA, IlvC, IlvE, SerA, and ThrL (Pulvermacher et al. 2008; Sharma et al. 2011). In E. coli, the expression of GcvB is intimately associated with the availability of glycine, and GcvB expression is induced when nutrients, especially glycine, are abundant in the environment. The $g c v B$ gene is adjacent to and transcribed divergently from $g c v A$, which encodes the GcvA protein that positively regulates both $g c v B$ and the glycine cleavage operon $g c v T H P$. The activation of both the $g c v T H P$ operon and $g c v B$, is repressed during growth in the absence of glycine due to the association between GcvA and the regulatory protein GcvR (Urbanowski et al. 2000). This interaction does not occur in the presence of glycine, leaving GcvA to act as an activator of $g c v B$ and $g c v T H P$ expression. Therefore, in E. coli and S. Typhimurium, during periods of low glycine abundance the decreased production of GcvB results in activation of the amino acid biosynthesis and transport proteins that are normally repressed by the GcvB sRNA (Urbanowski et al. 2000).

GcvB function has primarily been assessed in E. coli (Urbanowski et al. 2000; Pulvermacher et al. 2008; Coornaert et al. 2013), and S. Typhimurium (Sharma et al. 2011) with functional studies in other organisms limited to Yersinia pestis (McArthur et al. 2006). S. Typhimurium $g c v B$ mutants grow more slowly than the wild-type parent strain and E. coli $g c v B$ mutants have a decreased ability to form biofilms (Sharma et al. 2007; Mika and Hengge 2014). Analysis of the E. coli and S. Typhimurium GcvB mRNA targets has facilitated the identification of a GcvB binding sequence (seed region), 5'-CACAACAT- $3^{\prime}$, that allows for base pairing between $\mathrm{GcvB}$ and its mRNA targets. The mRNA seed region is strongly conserved in the GcvB targets produced by both species (Sharma et al. 2007). The seed region sequence, $5^{\prime}$-AUGUUGUG-3', is present in the GcvB expressed by both $S$. Typhimurium and E. coli (Sharma et al. 2011) and is the reverse complement of the seed region sequence present in the mRNA target molecules.

There is currently no information on the functional role of $\mathrm{GcvB}$, its mRNA targets, or mRNA binding interactions in any organisms from the Pasteurellaceae family. A bioinformatics screen of multiple genomes identified a putative GcvB homolog in P. multocida, and a recent bioinformatics analysis of the related organism, Actinobacillus pleuropneumoniae, also identified a GcvB homolog and its expression was confirmed by northern blotting (Sharma et al. 2007; Rossi et al. 2016). Another study in Haemophilus influenzae showed expression of GcvB was high when grown in the presence of primary normal human bronchial epithelial cells using RNA-seq (Baddal et al. 2015). However, neither study looked further into the function of GcvB. In this study, we report the characterization of $\mathrm{GcvB}$ in a highly pathogenic P. multocida strain and the identification of more than $30 \mathrm{tar}-$ gets. Furthermore, we identify the P. multocida GcvB seed region and use a two-plasmid green fluorescent protein (GFP) reporter system to confirm the binding interaction between P. multocida GcvB and one of its mRNA targets, gltA.

\section{RESULTS}

\section{Confirmation of GcvB expression in P. multocida using high-throughput transcriptomic analysis, northern blotting, and GcvB transcript analyses}

Previous bioinformatics analyses (Sharma et al. 2007) have identified a putative GcvB in P. multocida that contains the 


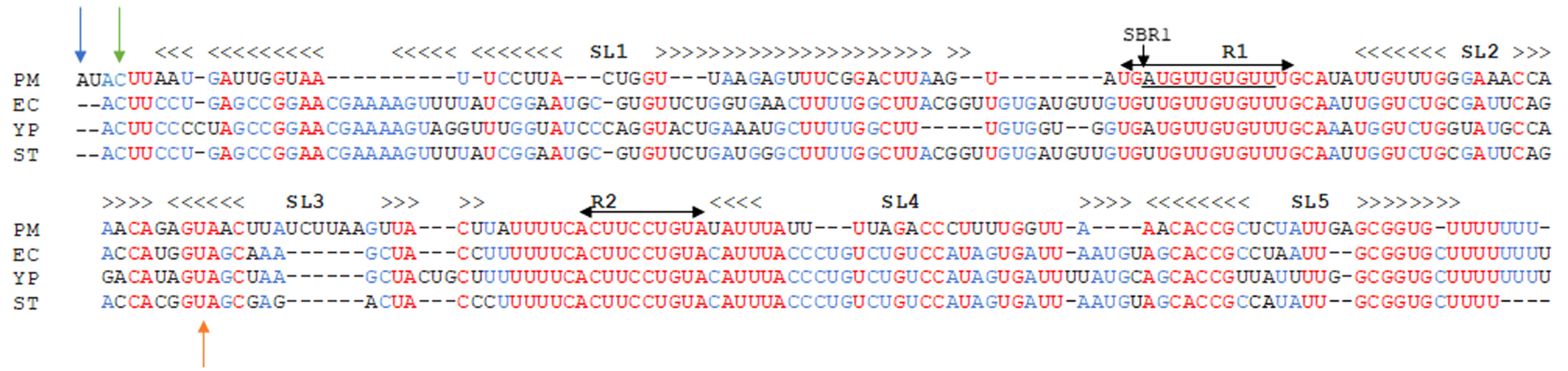

FIGURE 1. Nucleotide sequence alignment of GcvB from P. multocida (PM), E. coli (EC), Y. pestis (YP), and S. Typhimurium (ST). The previously identified S. Typhimurium GcvB stem-loop (SL) sequences (SL1-SL5), including their extent $(<$ and $>$ ) and conserved R1 and R2 sequences (horizontal arrows) (Sharma et al. 2007), are shown above the alignment. Nucleotides in red are identical across all four GcvB sequences; nucleotides in blue are identical in three of the four GcvB sequences. The proposed P. multocida seed region is labeled SBR1 and underlined. The predicted P. multocida GcvB rho-independent terminator sequence is labeled SL5. The orange arrow designates the position of the TargeTron intron insertion site in the P. multocida VP161 gcvB mutant. The green arrow indicates the predicted transcript start site for $P$. multocida GcvB as determined by primer extension. The blue arrow indicates the predicted start site for $P$. multocida GcvB as determined by $5^{\prime}$ RACE.

conserved R1 and R2 sequences common to all GcvB sRNA molecules (Fig. 1). The P. multocida gcvB was located between ivlE (encoding a branched-chain amino acid aminotransferase) and $g c v A$ (Fig. 2A). To determine if GcvB is expressed in $P$. multocida, we analyzed whole-transcriptome RNAsequencing (RNA-seq) data generated from RNA isolated from P. multocida VP161 grown until the cultures reached an optical density at $600 \mathrm{~nm}\left(\mathrm{OD}_{600}\right)$ of $0.2,0.7$, and 1.0 , representing early-exponential, mid-exponential and lateexponential growth phases in biological duplicate. The putative $P$. multocida GcvB sRNA was expressed strongly during early-exponential and mid-exponential growth, with an average of 1490 and 2514 GcvB transcripts per million (TPM) total transcripts, respectively (Fig. 2A). However, GcvB expression was reduced significantly by late-exponential growth (10-fold reduced expression compared to early exponential phase and 13-fold reduced expression compared to mid-exponential phase; false discovery rate $[\mathrm{FDR}]<0.01$ ) when only limited amounts of GcvB transcripts were produced (average of 209 GcvB TPM). The growth phase-dependent expression of the GcvB sRNA in P. multocida strain VP161 was confirmed by northern blotting using a GcvB complementary strand-specific, RNA probe. The probe hybridized strongly with a fragment of the predicted size ( $\sim 180 \mathrm{bp}$ ) of the GcvB sRNA transcript in the RNA isolated from VP161 cells in early-exponential growth phase, but only very weakly to RNA isolated from cells grown to lateexponential growth phase (Fig. 2B).
Analysis of the P. multocida GcvB sequence revealed a putative rho-independent transcriptional terminator that corresponded to stem-loop 5 (SL5) present in the GcvB of E. coli and S. Typhimurium (Fig. 1; Sharma et al. 2007). The position of this putative stem-loop corresponded closely with the end of the RNA-seq transcript peak (Fig. 2A) and we predict that this stem-loop defines the $3^{\prime}$ end of the P. multocida GcvB. To determine the $5^{\prime}$ end of the $g c v B$ transcript, two independent methods were used, primer

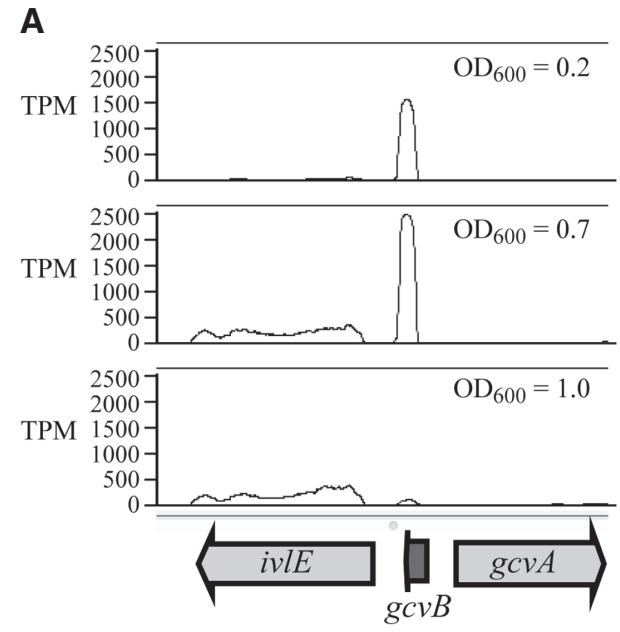

FIGURE 2. GcvB is expressed strongly at early- and mid-exponential growth phases but only weakly at late-exponential growth phase. $(A)$ Number of mapped transcripts per million (TPM) total transcripts that map to the genomic regions surrounding $g c v B$ as determined by whole-genome RNA-seq and as visualized in Artemis (Sanger) genome viewer. The top panel shows the number of mapped reads using RNA derived from early-exponential growth phase $\left(\mathrm{OD}_{600}=0.2\right)$ cells, the middle panel shows the number of mapped reads using RNA derived from mid-exponential growth phase $\left(\mathrm{OD}_{600}=0.7\right)$ cells, and the bottom panel shows the number of mapped reads using RNA derived from late-exponential growth phase $\left(\mathrm{OD}_{600}=1.0\right)$ cells. The extent and orientation of the genes $i v l E, g c v B$, and $g c v A$ are shown below the mapping graphs. $(B)$ P. multocida RNA $(8 \mu \mathrm{g} / \mathrm{lane})$ isolated from early-exponential growth phase $\left(\mathrm{OD}_{600}=0.2\right)$ cells and late-exponential growth phase $\left(\mathrm{OD}_{600}=1.0\right)$ cells was used for northern blotting together with a DIG-labeled, single-stranded RNA probe representing the sequence complementary to GcvB. The position of GcvB is shown at the left. Image has been modified to increase contrast. 
extension and $5^{\prime}$ RNA ligase-mediated rapid amplification of cDNA ends ( $5^{\prime}$ RLM-RACE). For primer extension experiments, RNA isolated from $P$. multocida VP161 was used as the template for CDNA synthesis with the fluorescently labeled primer BAP7962 or primer BAP8190 (Supplemental Table S1) that anneal $\sim 90 \mathrm{bp}$ and $\sim 140 \mathrm{bp}$, respectively, from the predicted start of the $g c v B$ transcript (as determined by the RNA-seq transcriptomic analyses). Fragment size analysis of the generated cDNA molecules identified a fragment of $87 \mathrm{nt}$ in length for the primer extension using BAP7962 and $140 \mathrm{nt}$ in length for primer extension using BAP8190. These data indicated that the P. multocida VP161 GcvB transcript started with the sequence 5'-CUUAAUG$3^{\prime}$, plus or minus the $5^{\prime} \mathrm{C}$, which corresponds to the second nucleotide in the GcvB sequence from E. coli and S. Typhimurium (Fig. 1). To determine if this was a bona fide transcript initiation site, we used $5^{\prime}$ RLMRACE. P. multocida VP161 RNA was first treated with calf intestine alkaline phosphatase and tobacco acid pyrophosphatase and then used as the template in nested PCRs to generate $5^{\prime}$ adapter-ligated GcvB DNA fragments, which were then cloned into the plasmid pCR2.1. DNA sequencing of these cloned fragments using a vector-specific primer (BAP612) revealed that the P. multocida GcvB transcriptional start site was located 2 bp upstream of the E. coli and S. Typhimurium GcvB start sites (Fig. 1; Supplemental Fig. 1). Therefore, these data indicate that the P. multocida GcvB transcript begins with 5'-AUACUUAAUG-3'.

The secondary structure of the P. multocida GcvB was modeled (Fig. 3A) using the Mfold webserver (Zuker 2003). While the predicted structure is very similar to the experimentally determined structure of the $S$. Typhimurium GcvB (Sharma et al. 2007), there are some notable differences. These include the observation that the stem-loop 1 (SL1) in the P. multocida GcvB is predicted to be significantly shorter than the SL1 in the $S$. Typhimurium GcvB; sequence alignment of the GcvB sRNAs from P. multocida, E. coli, S. Typhimurium and $Y$. pestis confirmed that the $5^{\prime}$ region of the P. multocida GcvB is indeed shorter (Fig. 1). The predicted P. multocida GcvB structure contains the SL2 and SL3 stem-loops between the conserved $\mathrm{R} 1$ and R2 G/U-rich linker regions, as is observed in the $S$. Typhimurium GcvB.
However, the P. multocida GcvB has no predicted SL4 stem-loop, but rather the region between SL1 and R1 shows high complementarity to the region between R2 and SL5 and may form a long double-stranded section, although this remains to be experimentally verified (Fig. 3A).

\section{GcvB predominately regulates amino acid biosynthesis and transport proteins in $P$. multocida}

In order to determine the GcvB regulon in P. multocida, a VP161 $g c v B$ mutant (AL2677) (Supplemental Table S2) was constructed using TargeTron technology (Sigma-Aldrich). The intron insertion was located between nucleotides 92 and 93 of $g c v B$ and within the predicted SL3 loop (Fig. 1,

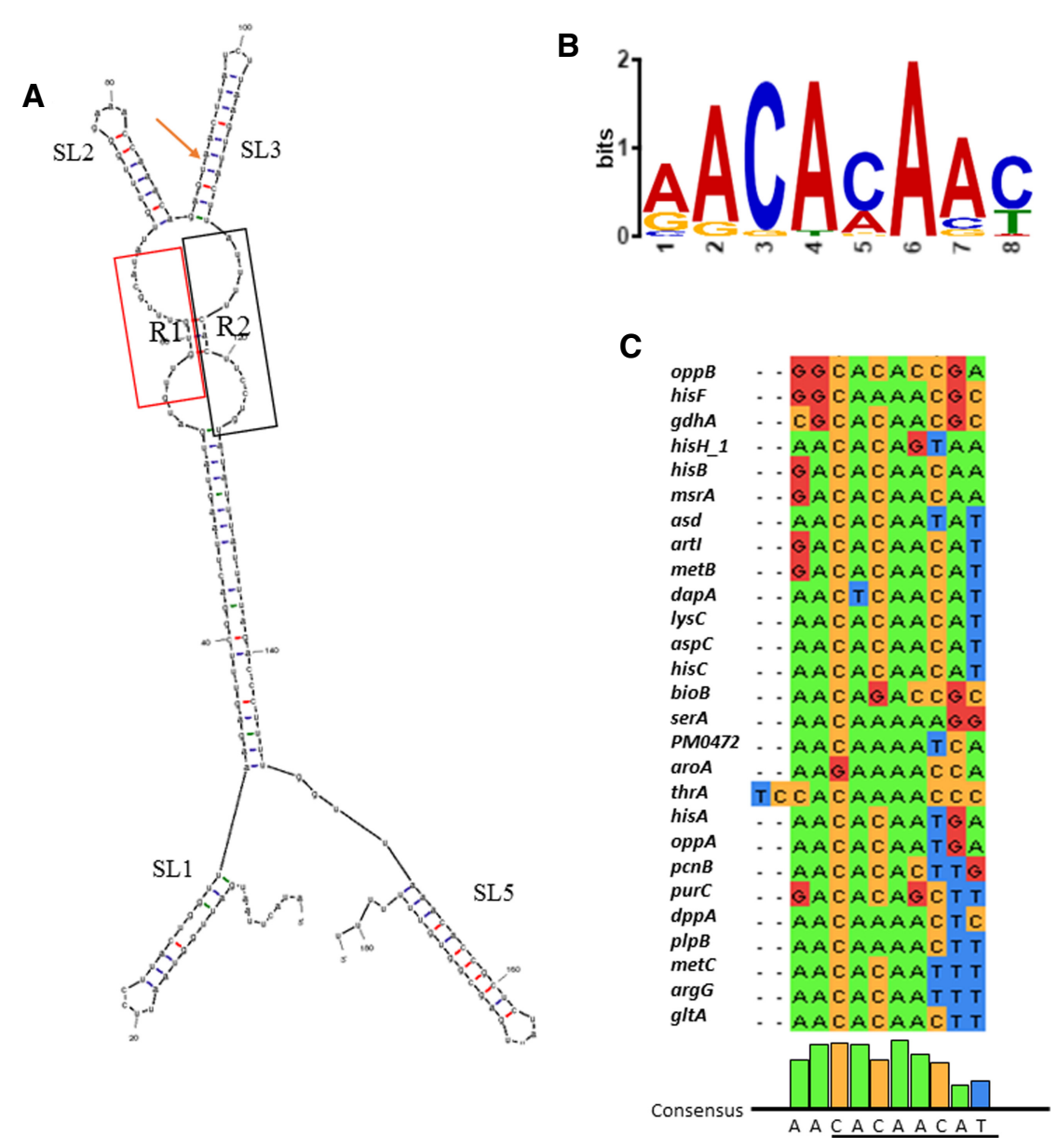

FIGURE 3. Predicted secondary structure of the P. multocida GcvB and the seed binding consensus motifs present in the 27 putative mRNA targets. $(A)$ Putative secondary structure of the $P$. multocida GcvB sRNA molecule as predicted by Mfold. The conserved R1 (red) and R2 (black) sequences are boxed and the proposed SL1, SL2, SL3, and SL5 stem-loops are labeled. The position of the TargeTron intron in the P. multocida $g c v B$ mutant is also shown (orange arrow). (B) Diagram of the GcvB mRNA target seed binding motif identified by MEME in 27 genes encoding putative GcvB mRNA targets. The letter height indicates the frequency of each base at each position. $(C)$ Sequence alignment of the 27 putative seed binding regions found by MEME motif finder in genes encoding the predicted GcvB mRNA targets. Nucleotides are highlighted with color as follows to show the level of conservation: (A) green; (C) yellow; (T) blue; and (G) red. The $P$. multocida GcvB consensus sequence is shown beneath the alignment, with the E. coli and S. Typhimurium core GcvB-mRNA seed binding sequence underlined (Sharma et al. 2007). 
Fig. 3A). To complement the mutation, the wild-type VP161 $g c v B$ gene, together with its putative native promoter, was cloned into the P. multocida plasmid pPBA1100s to generate the plasmid pAL1190 (Supplemental Table S2). This plasmid was used to transform the P. multocida gcvB mutant AL2677, producing the strain AL2864 (Supplemental Table S2). As a control, pPBA1100s empty vector (Supplemental Table S2) was also used to transform the $g c v B$ mutant, generating the strain AL2862.

The level of $g c v B$ expression in these strains was determined using qRT-PCR with all expression levels normalized to the expression of the housekeeping gene gyrB (Fig. 4). The levels of $g c v B$ expression in the wild-type strain (normalized to $g y r B)$ were $0.24 \pm 0.04(n=3$, SEM $)$ and $0.13 \pm 0.03$ at early- and mid-exponential growth phases, respectively. As expected no expression of $g c v B$ was measured in the $g c v B$ mutant at either growth phase, as the primers used for the qRT-PCR spanned the point of the relatively large intron insertion. Surprisingly, the levels of $g c v B$ expression in AL2864 ( $g c v B$ mutant provided with an intact copy of $g c v B$ on the plasmid pAL1190) were $16.4 \pm 2.5$ and $37.6 \pm 16.4$, indicating a 69-fold increase in $g c v B$ expression at early-exponential growth phase and a 289-fold increase at mid-exponential growth phase compared to expression in the wild-type strain. Thus, providing the $g c v B$ mutant with functional $g c v B$ in trans resulted in the significant overexpression of $\mathrm{GcvB}$ at both growth phases tested. Accordingly, the strain AL2864 was designated as a GcvB overexpression strain. It was predicted that that there would be an inverse relationship between the levels of expression of any GcvB-regulated genes in the $g c v B$ overexpression strain and the levels of expression of the same genes in the $g c v B$ deficient strains $(g c v B$ mutant

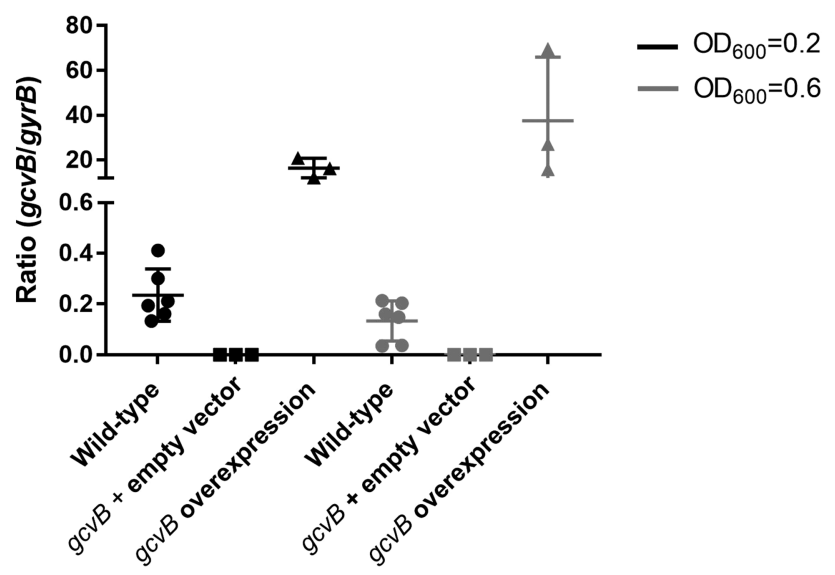

FIGURE 4. qRT-PCR analyzing the expression of GcvB in wild-type $P$. multocida expression (represented by circles), the $g c v B$ mutant with empty vector (square symbols), and the GcvB overexpression strains (triangle symbols). RNA was isolated from early exponential growth phase cells $\left(\mathrm{OD}_{600}=0.2\right)$ (black symbols) and mid-exponential growth phase cells $\left(\mathrm{OD}_{600}=0.6\right)$ (gray symbols). Expression was standardized to the expression of the housekeeping gene gyrB. Thick horizontal bars represent the mean $\pm \mathrm{SD}$. alone, or $g c v B$ mutant containing empty vector). To test this, the strain was included in the proteomic analyses described below.

The survival and growth of the wild-type VP161, the GcvB-deficient strains (AL2677 and AL2862) and the GcvB overexpression strain (AL2864) was examined under several conditions. It was found that the $g c v B$ mutant strains grew indistinguishably from the wild-type VP161 during growth in heart infusion (HI) broth (Fig. 5A,B). The $g c v B$ overexpression strain had a similar exponential growth rate (doubling time of $41.1 \pm 0.5 \mathrm{~min}$ ) to the wild-type strain (doubling time of $36.2 \pm 2.1 \mathrm{~min}$ ); however, the lag-phase was increased by $\sim 1.5 \mathrm{~h}$ (Fig. $5 \mathrm{~B}$ ). There was no difference in the ability of the $g c v B$ mutant, AL2677, and the wild-type VP161 to form biofilms during static growth (Fig. 5C) and no difference in survival at low $\mathrm{pH}$ (HI broth, $\mathrm{pH}=4.6$ for $15 \mathrm{~min}$ ) (Fig. 5D).

We then analyzed the protein expression profiles of the wild-type VP161, $g c v B$ mutant (AL2677), $g c v B$ mutant plus empty vector (AL2862), and GcvB overexpression strain (AL2864) using liquid chromatography coupled to tandem mass spectrometry (LC-MS/MS) in biological triplicate. The initial experiment compared wild-type VP161 and the $g_{c v B}$ mutant using isotopically labeled samples. The second experiment compared wild-type, $g c v B$ mutant plus empty vector and $\mathrm{GcvB}$ overexpression strain using label-free proteomics. For all experiments, cells were harvested at early-exponential growth phase when $g c v B$ is strongly expressed in the wild-type strain. In total, 1191 proteins were identified in the first experiment (using isotopic labeling) and 1540 proteins in the second (label-free); representing 57\% and $74 \%$, respectively, of the 2085 total proteins predicted to be encoded on the P. multocida VP161 genome (Boyce et al. 2012). Identified proteins were considered differentially expressed if they showed $a \geq 1.5$-fold $\left(\geq 0.59 \log _{2}\right)$ difference in production at an FDR of $<0.05$ compared to wild-type VP161. Overall 36 proteins were measured as showing increased production in either of the two $g c v B$ mutant strains analyzed; 25 proteins in experiment 1, 28 in experiment 2 with 17 identified in both experiments (Supplemental Table S3). Only 10 proteins showed decreased production in either of the two $g c v B$ mutant strains analyzed; two in experiment 1 , eight in experiment 2 with none identified in both experiments (Supplemental Table S4). In contrast, 218 proteins with altered production levels were identified in the GcvB overexpression strain, 75 with increased production, and 143 with decreased production (Supplemental Tables S5, S6).

We then compared the lists of differentially produced proteins identified in each of the GcvB-deficient strains $(g c v B$ mutant strain AL2677, and $g c v B$ mutant plus empty vector, AL2862) and the GcvB overexpression strain. A total of 27 proteins showed significantly increased production in one of the GcvB-deficient strains as well as inverse (decreased) production in the $g c v B$ overexpression strain (Table 1). Of these 27 proteins, 17 proteins $(71 \%)$ displayed increased 


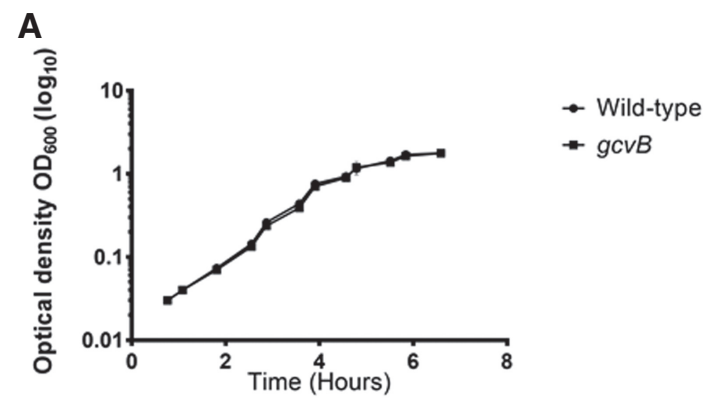

B
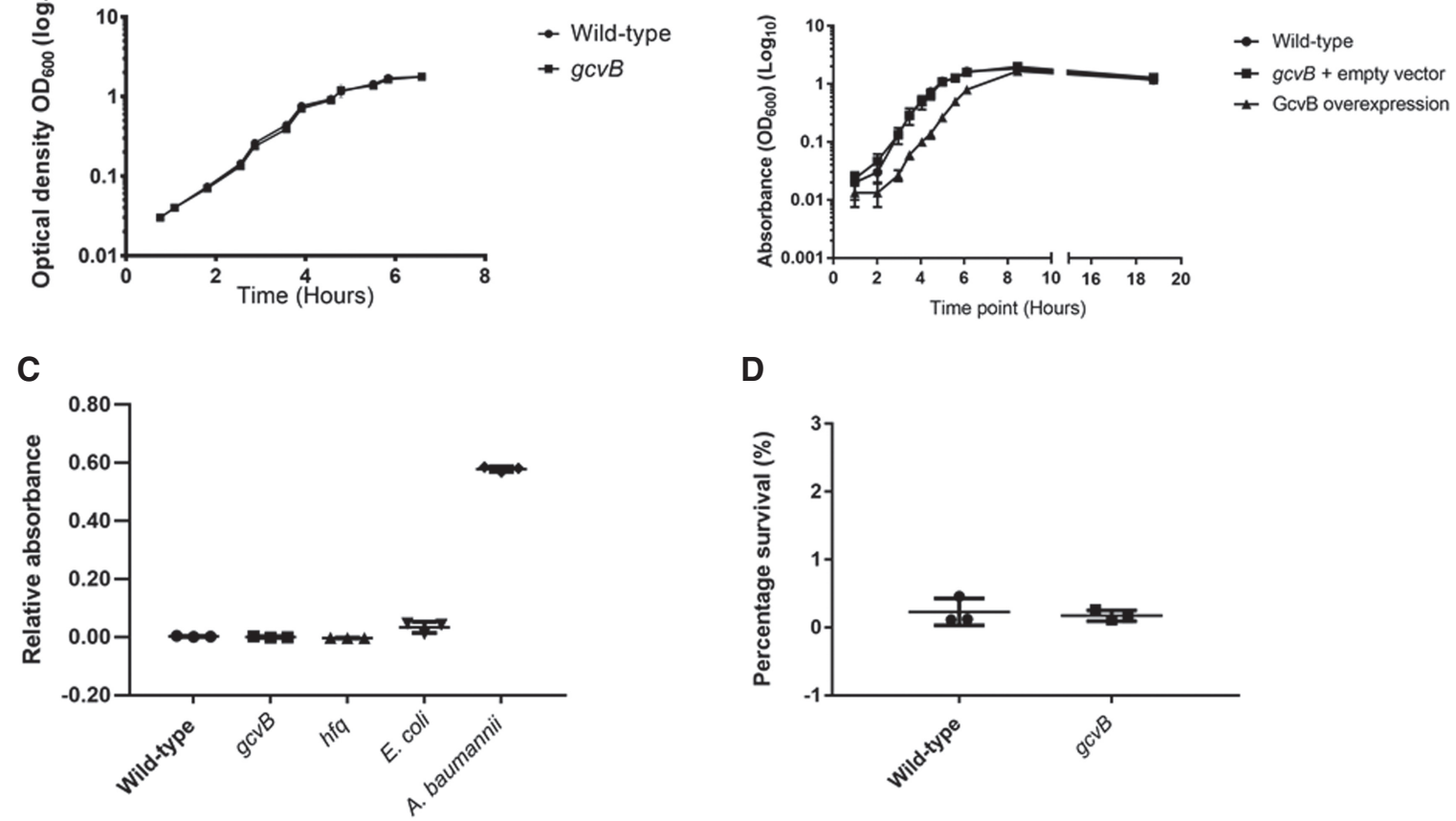

D

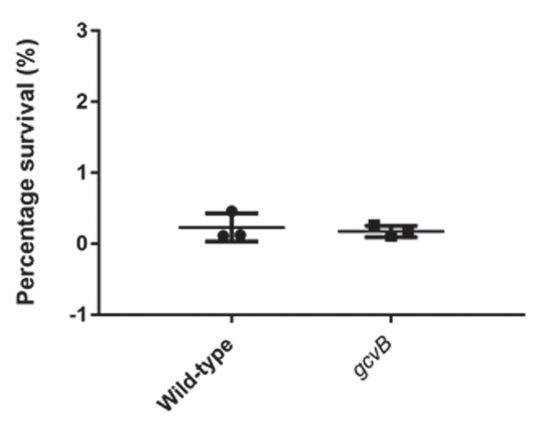

FIGURE 5. (A) Growth curve of wild-type P. multocida VP161 (circles) and the $g c v B$ mutant strain (squares) in Heart infusion broth, incubated with shaking at $37^{\circ} \mathrm{C}$ for $7 \mathrm{~h}$. Data shown are mean $\pm \mathrm{SD}(n=3)$. (B) Growth curve of wild-type P. multocida VP161 (circles), gcvB mutant containing empty vector (squares), and the $g c v B$ overexpression strain (triangles) grown for $24 \mathrm{~h}$ under the same conditions as above. (C) Relative absorbance compared to a no bacteria control, observed following a static crystal violet biofilm assay. The P. multocida wild-type VP161 (circles) was compared to the gcvB mutant strain (squares) and the $h f q$ mutant strain (upright triangles). Controls included $E$. coli (upside-down triangles), an intermediate biofilmforming species, and A. baumannii (hexagons), a strong biofilm-forming species. Horizontal lines represent mean \pm SD $(n=3)$. (D) Survival of $P$. multocida wild-type VP161 (circles) and the gcvB mutant strain (squares) following $15 \mathrm{~min}$ of acid stress at pH 4.6. Horizontal lines represent mean $\pm \mathrm{SD}(n=3)$.

production in both of the GcvB-deficient strains analyzed (Table 1). A total of 10 proteins showed decreased production in one of the GcvB-deficient strains (AL2677 or AL2862), but none of these showed decreased production in both GcvB-deficient strains and only one (Tpl) showed inverse (increased) production in the $g c v B$ overexpression strain.

\section{P. multocida GcvB binds Hfq and many of the GcvB mRNA targets are unique}

The binding of GcvB with many mRNA targets in E. coli and $S$. Typhimurium is known to be Hfq-dependent. To confirm P. multocida GcvB bound to Hfq we expressed a FLAG-tagged Hfq in P. multocida and used coimmunoprecipitation followed by high-throughput sequencing to identify precipitated RNAs (in triplicate samples). Sequences matching GcvB were recovered from the FLAG-tagged Hfq samples at high numbers, on average 774.7 reads per sample, but at significantly reduced numbers in the untagged control sample, an average of 39.3 reads per sample $(\mathrm{FDR}<0.05)$. Therefore, we conclude that $P$. multocida GcvB can bind P. multocida Hfq. Given this information, the list of proteins identified as differentially produced in the P. multocida $g c v B$ mutant analyses was compared to the list of proteins identified as differentially produced in the previously analyzed $P$. multocida $h f q$ mutant (Mégroz et al. 2016). Ten of the proteins that showed increased production in the P. multocida $h f q$ mutant also showed increased production in both of the GcvB-deficient strains and a further five showed increased production in one of the GcvB-deficient strains (AL2677 or AL2682). These proteins were Asd, DapA, DppA, GdhA, GltA, HisC, HisH_1, IlvG, LysC, MetB, OppA, PlpB, PurC, RcpA, and RsgA_2 (Supplemental Table S3). One protein, SpeF, showed decreased production in the $h f q$ mutant at mid-log growth phase (Mégroz et al. 2016) but increased production in the gcvB mutant, AL2677 (Supplemental Table S3). The list of proteins with altered production in the P. multocida GcvBdeficient strains was also compared with the 54 GcvBregulated targets identified in E. coli and $S$. Typhimurium (Sharma et al. 2011). Of these 54 known targets, 42 had homologs in the P. multocida genome and all but five of these were measured in our proteomics experiments. However, only eight were identified as differentially produced in our proteomics experiments; namely, DppA, GdhA, LysC, OppA, OppB, PlpB, SerA, and ThrA (Supplemental Table S3). 


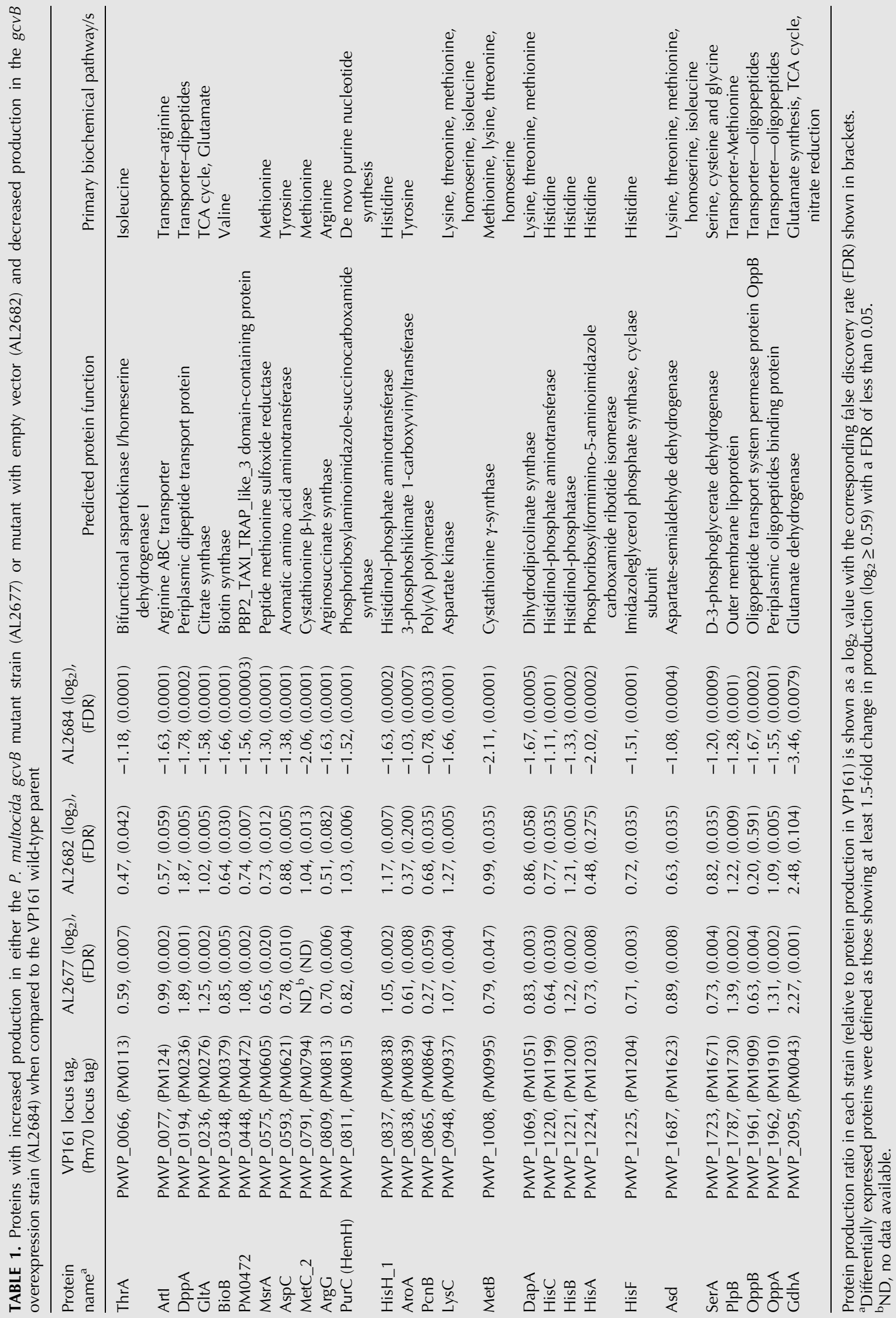




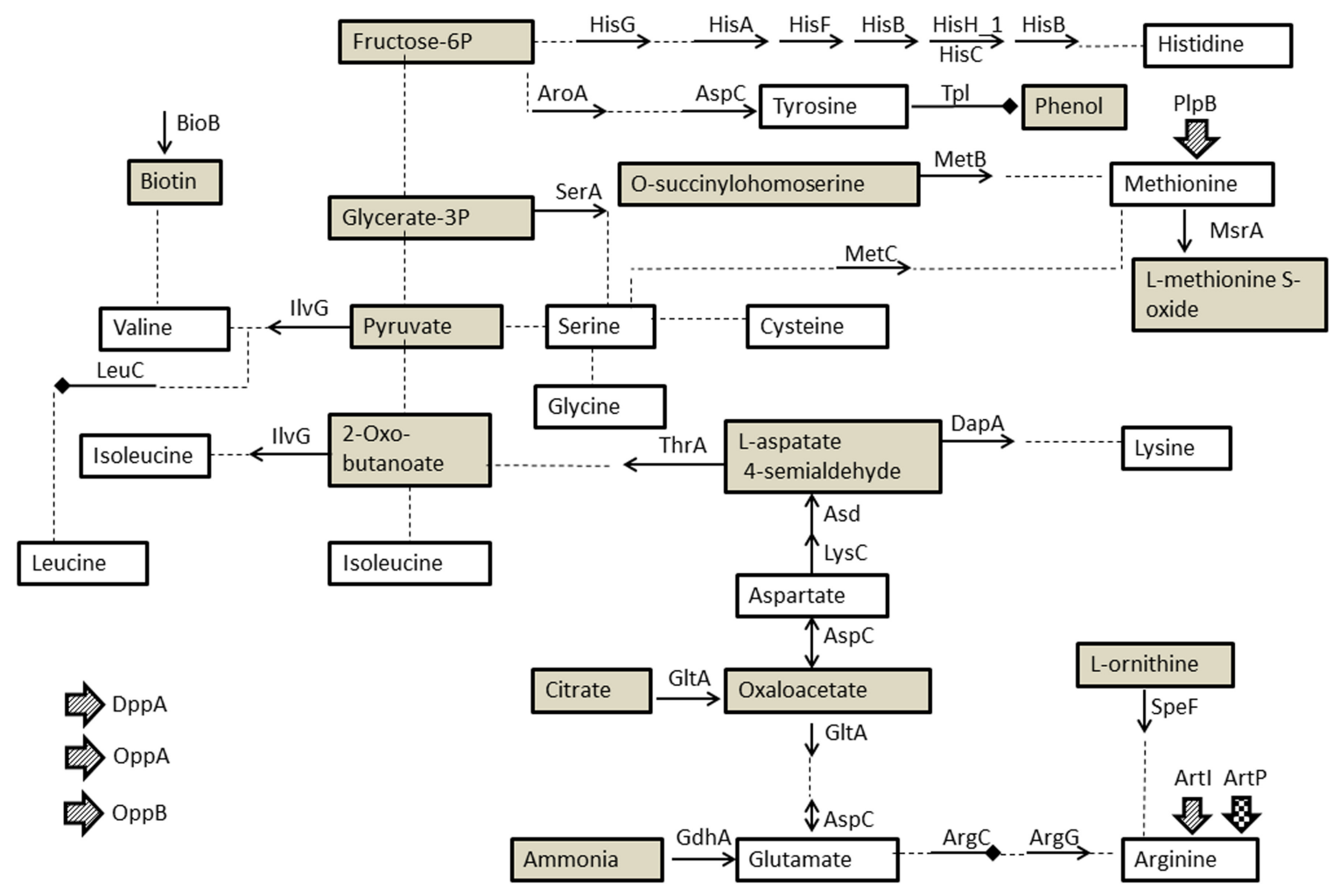

FIGURE 6. Amino acid biosynthesis pathways predicted to be affected by $g c v B$ inactivation in $P$. multocida strain VP161. The amino acids whose biosynthesis is predicted to be regulated by GcvB are within white boxes. Amino acid biosynthesis proteins whose production is negatively regulated by $\mathrm{GcvB}$ are shown at the relevant pathway step with open-headed arrows. Proteins whose production is positively regulated by $g c v B$ are indicated at the relevant pathway step with closed diamond-headed arrows. Large, diagonally striped, and checked arrows indicate predicted amino acid or oligo peptide transport proteins that are negatively and positively regulated by $g c v B$, respectively.

The proteins identified as differentially produced (increased or decreased production) in P. multocida following inactivation of $g c v B$ in either experiment (Supplemental Tables S3, S4), were mapped to their metabolic pathways; amino acid biosynthesis proteins were observed to be highly overrepresented (Fig. 6; Fishers exact test; $P<10^{-11}$ ). These amino acid biosynthesis proteins included 21 with increased production (ArgG, AroA, Asd, AspC, BioB, DapA, GdhA, GltA, HisH_1, HisA, HisB, HisC, HisF, HisG, IlvG, LysC, MetB, MetC_2, SerA, SpeF, and ThrA) and three with decreased production (ArgC, LeuC, and $\mathrm{Tpl}$ ). Furthermore, another five proteins with increased production were predicted to be involved in the transport of amino acids or oligopeptides (ArtI, DppA, OppA, OppB, and PlpB), as well as one protein with decreased production (ArtP). Thus, 27 of the 36 proteins negatively regulated by $\mathrm{GcvB}$ are involved in biosynthesis or transport of at least 14 different amino acids (Fig. 6). Moreover, of the 17 proteins that displayed increased production in both GcvB-deficient strains and an equivalent decrease in the $g c v B$ overexpression strain, only PurC (predicted to be involved in de novo purine biosynthesis), and PM0472 (an uncharacterized periplasmic binding protein containing a PBP2_TAXI_TRAP_like_3 domain), were not predicted to be involved in amino acid transport and metabolism.

\section{Bioinformatic analyses identify an extended GcvB seed region binding motif}

In order to determine if each of the experimentally identified putative P. multocida GcvB targets contained a conserved region that may serve as a GcvB binding site, the DNA sequence starting $120 \mathrm{nt}$ upstream of the start codon and continuing to $60 \mathrm{nt}$ downstream from the start codon of each gene was examined for conserved sequence motifs using the Multiple Em for Motif Elicitation (MEME) tool (Bailey et al. 2009). Initially, all of the genes encoding the proteins identified as differentially produced following inactivation of $g c v B$ (i.e., all proteins in Supplemental Tables S3 and S4) were examined. However, this analysis failed to identify a conserved motif across all proteins. We then constrained the target list to include only those proteins that showed increased differential production in either of the $g c v B$ mutant strains and had a corresponding inverse production in the $g c v B$ overexpression strain (Table 1$)$. Using the DNA sequences $(-120$ to $+60 \mathrm{nt})$ of these genes, a consensus sequence consisting of $5^{\prime}$-AACACAAC-3' $\left(E\right.$-value: $\left.3.2 \times 10^{-7}\right)$ was identified in all targets (Fig. 3B). The sequences around this identified motif were also aligned using Clustal Omega (Sievers et al. 2011), which revealed a highly conserved slightly extended 


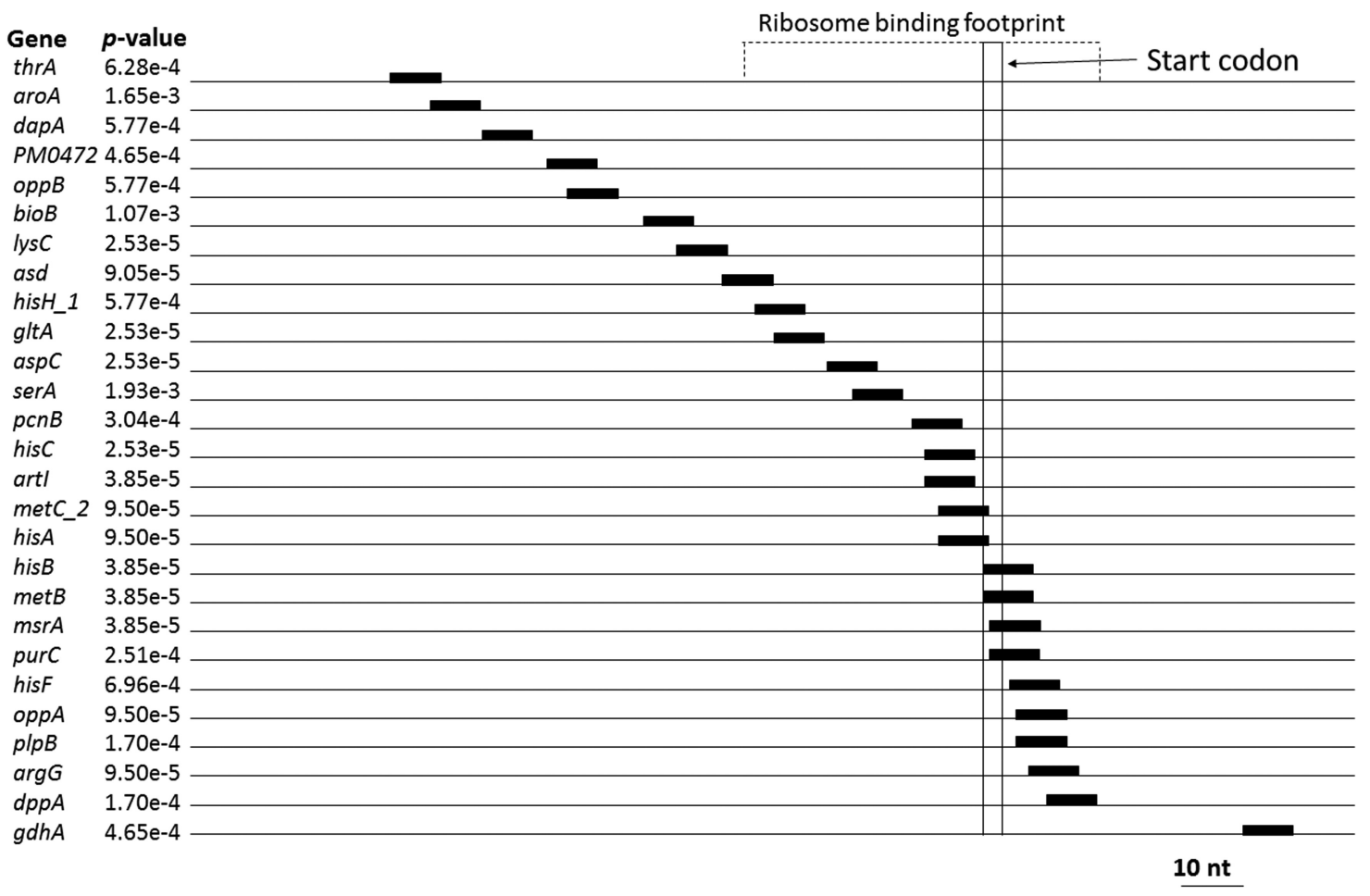

FIGURE 7. Schematic representation of the position of the predicted GcvB seed binding regions (black boxes) in 27 GcvB mRNA targets (listed), relative to the start codon. The $P$-values, generated by MEME motif finder, are shown at the left and give the likelihood of each identified motif occurring in the analyzed sequence fragment by chance. The predicted ribosome footprint is indicated by the dashed line (top). A scale bar is shown at the bottom.

consensus sequence (5'-AACACAACAT-3') (Fig. 3C). Therefore, we predict that the P. multocida GcvB seed binding sequence is slightly longer than the E. coli and $S$. Typhimurium GcvB seed region, but that the eight central nucleotides (5'-CACAACAT-3') are identical. Importantly, the reverse complement of the extended $P$. multocida GcvB seed sequence, $5^{\prime}$-AUGUUGUGUU- $3^{\prime}$, is present within the sequence of GcvB; this sequence was identical to the same region in the $Y$. pestis $\mathrm{GcvB}$ and differed by just a single nucleotide compared to the same region in the E. coli and S. Typhimurium GcvB (Fig. 1). The position of this consensus GcvB binding sequence was then mapped on each of the 27 mRNA targets (Fig. 7). The binding sequence was located upstream of the predicted ribosome binding footprint [ -39 to +19 bp (Hüttenhofer and Noller 1994; Sharma et al. 2007)] in seven mRNA targets and was overlapping, or within the ribosome binding footprint, in 19 targets. In one target, $g d h A$, the binding sequence was downstream from the ribosome binding footprint (Fig. 7). As four of the identified GcvB targets were known GcvB targets in Salmonella and E. coli, the putative seed binding regions of the P. multocida targets were compared to the known GcvB seed binding regions in $о p p A, d p p A$, serA, and $g d h A$ encoded by Salmonella (Sharma et al. 2007, 2011). It was found that the seed region for the $P$. multocida oppA was located at the same position relative to the seed region of $o p p A$ in Salmonella and contained a similar sequence (Sharma et al. 2007). The seed region for the $P$. multocida serA was located close to the seed region position reported for serA in Salmonella but the sequence was dissimilar (Sharma et al. 2011). In contrast, the predicted seed regions for $P$. multocida $d p p A$ and $g d h A$ were found in different locations to those reported for the equivalent genes in Salmonella and had only limited sequence similarity (Sharma et al. 2007, 2011).

In order to determine if the identified GcvB seed region was present in all mRNAs encoding proteins predicted to be regulated by $G c v B$, the corresponding DNA sequences $(-120$ to $+60 \mathrm{nt})$ were visually inspected. Two more seed sequences were identified that exactly matched the consensus sequence generated with MEME, and these were located in $g l p Q$ and $l e u C$, positioned at 34 and 0 nt upstream of the start codon, respectively. The remaining putative targets had no sites with less than one or two mismatched nucleotides at critical positions ( 3 and 6 ).

\section{Modification of a two-plasmid GFP reporter system to detect $P$. multocida sRNA-mRNA interaction in $E$. coli}

In order to experimentally confirm that the conserved sequence $5^{\prime}$-AACACAACAT- $3^{\prime}$ contained the $P$. multocida GcvB seed region, sRNA/mRNA interaction experiments using two recombinant plasmids were conducted in E. coli 
strain $\mathrm{DH} 5 \alpha$, based on a previously described two-plasmid GFP reporter system (Urban and Vogel 2007). P. multocida Hfq shares $92.7 \%$ identity with two-thirds of the E. coli Hfa protein (amino acids 1-73) but shares only $13.7 \%$ identity with the C-terminal region of E. coli Hfq (amino acids 74-102). Therefore, before using this system, we first assessed whether E. coli Hfq could act as a chaperone for P. multocida sRNA molecules. A P. multocida expression plasmid containing a functional copy of the E. coli DH5a hfq (pAL1266, Supplemental Table S2) was used to transform the P. multocida VP161 hfq mutant, which produces only low levels of hyaluronic acid capsule compared to the parent strain VP161 (Mégroz et al. 2016). When the P. multocida hfq mutant was complemented with pAL1266 (expressing E. coli $h f q$ ), capsule production was restored to the same level as that observed when the $h f q$ mutant was complemented with the native $P$. multocida $h f q$ gene (Fig. 8 ). Thus, these data show that the native E. coli Hfq molecule can appropriately chaperone $P$. multocida sRNAs, allowing $E$. coli to be used as the host cell for the P. multocida sRNA-mRNA interaction studies described below.

To produce a two-plasmid GFP reporter system for our experiments, two expression vectors were constructed, designated pREXY and pTEXY (Supplemental Table S2). The pREXY plasmid is a shuttle vector used for the expression of P. multocida sRNAs (GcvB in this case) in either E. coli

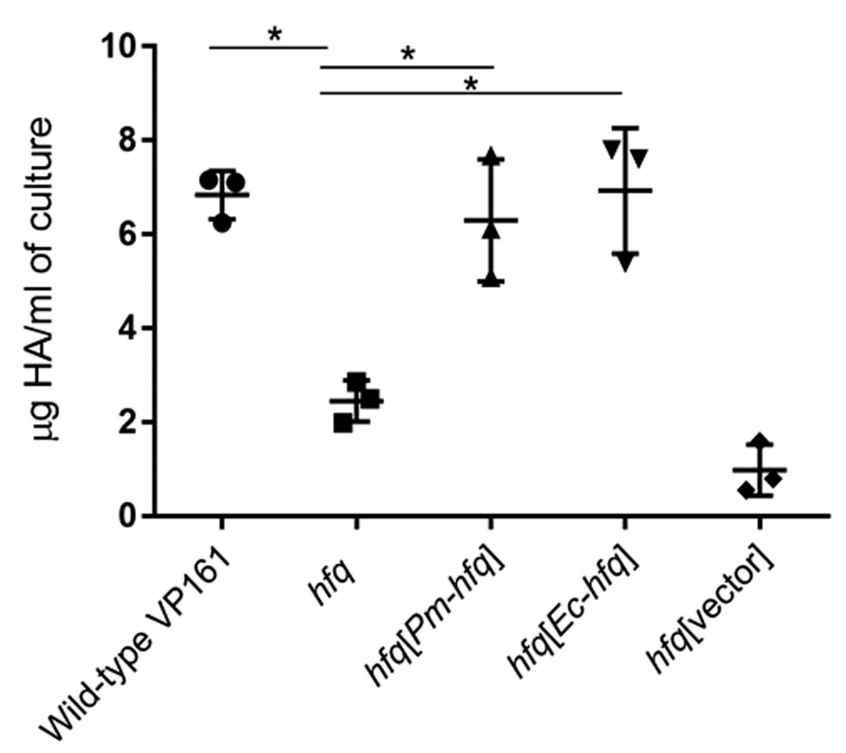

FIGURE 8. Hyaluronic acid capsule production in the P. multocida $h f q$ mutant containing a functional copy of $h f q$ from E. coli or P. multocida. The amount of hyaluronic acid capsular material produced during midexponential growth by P. multocida wild-type strain VP161, P. multocida $h f q$ mutant $(h f q), P$. multocida $h f q$ mutant complemented with a functional copy of the native $h f q$ from P. multocida ( $h f q[P m-h f q]$ ), $P$. multocida $h f q$ mutant containing a functional copy of the $h f q$ from E. coli ( $h f q[E c-h f q])$, or the P. multocida $h f q$ mutant containing empty vector ( $h f q[$ vector]). Each data point shows a single hyaluronic acid measurement. Thick horizontal bars represent the mean and error bars show \pm 1 SD $(n=3)$. (*) $P<0.05$ using Student's $t$-test. or P. multocida and contains a $P$. multocida tpi promoter upstream of the multiple cloning site (MCS). The second plasmid, pTEXY, is used for the transcriptional and translational coupling of the mRNA target with superfolder GFP (sfGFP) under the control of the tetracycline promoter $\left(P_{\text {tet0-1 }}\right)$.

\section{GcvB inhibits GltA production via complementary binding between the predicted seed regions in GcvB and gltA}

For recombinant expression of $P$. multocida $g c v B$ sRNA in E. coli, the entire $g c v B$ gene from $P$. multocida strain VP161 was PCR-amplified and cloned into the MCS of pREXY, generating the GcvB expression plasmid pAL1197. For recombinant expression of a predicted $g c v B$ target region, a $P$. multocida fragment containing $38 \mathrm{bp}$ upstream and the first $60 \mathrm{bp}$ of gltA was cloned into the XbaI and BglII sites of pTEXY, located between $P_{\text {tet0-1 }}$ and sfGFP to produce a $g l t A-s f G F P$ translational fusion. This plasmid was named pAL1257 (Supplemental Table S2). The recombinant plasmids, or vector only, were used in various combinations to transform competent E. coli DH5a. Restriction digest analysis and DNA sequencing confirmed all transformants contained the correct plasmids.

The E. coli strain containing both the pTEXY::gltA-sfGFP expression plasmid and the empty pREXY vector (no GcvB) was highly fluorescent, but the strain containing both the pTEXY::gltA-sfGFP plasmid and the pREXY::gcvB expression plasmid showed significantly reduced fluorescence $(P<0.0005$; Fig. 9). Thus, expression of GcvB represses production of the GltA-sfGFP fusion protein, as would be expected for a bona fide GcvB target mRNA.

In order to confirm that the GcvB-mediated repression of GltA expression was specifically due to complementary base pairing between the predicted seed regions, two modified plasmids were constructed and tested for fluorescence in the two-plasmid GFP reporter system. Firstly, the putative central seed sequence in the gltA upstream region was replaced with a nucleotide sequence identical to the central seed region of the GcvB sRNA (UGUGUUG) to generate the plasmid pTEXY::gltA $A_{M S R 1}-s f G F P$ (pAL1290; Supplemental Table S2). The E. coli strain containing this plasmid, with the gltA seed region mutation, and the pREXY:: $g c v B$ plasmid showed levels of fluorescence indistinguishable from the fluorescence of the strains containing pTEXY::gltA-sfGFP and empty pREXY (no GcvB). This indicates that GcvB was unable to repress the production of GltA following the mutation of the gltA seed region. Secondly, the plasmid pREXY:: gcvB $B_{M S R 2}$ (pAL1277; Supplemental Table S2) was generated, encoding a modified $g c v B$ that contained a nucleotide sequence identical to the seed region of glt $A$ mRNA target (ACACAAC), instead of the GcvB seed region (UGUGUUG). The E. coli strain containing both this plasmid and the pTEXY::gltA-sfGFP showed levels of fluorescence indistinguishable from the fluorescence of the strains 


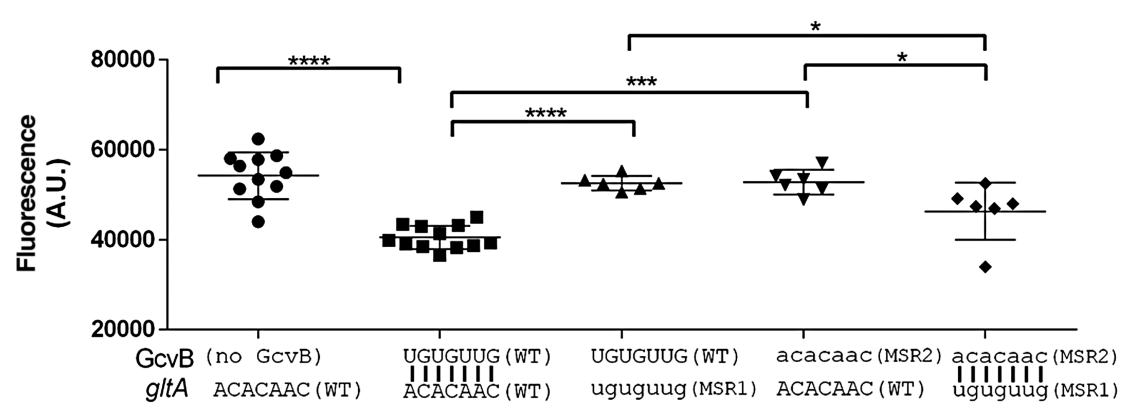

FIGURE 9. Superfolder green fluorescent protein (sfGFP) production in E. coli strains containing different plasmid pairs. Each E. coli strain harbored one pREXY sRNA expression plasmid derivative and one pTEXY mRNA::sfGFP reporter derivative. The top line of the $x$-axis label shows the sequence of the native (WT) or mutated (MSR2) seed region within the recombinant $g_{c v B}$ in the plasmid pREXY::gcvB or pREXY:: $g c v B_{\mathrm{MSR} 2}$, respectively. A pREXY vector only control (no GcvB) was also included in the study. The bottom line of the $x$-axis shows the sequence of the native (WT) or mutated (MSR1) seed region within the recombinant gltA fused to the sfGFP gene in the pTEXY::gltA-sfGFP or pTEXY::glt $A_{\mathrm{MSR} 1}-s f G F P$, respectively. Wild-type seed sequence is shown in all capitals, mutated seed sequence is shown in lower case. Vertical lines between the text show if complementary base pairing is predicted between the sRNA and mRNA seed sequence. The amount of sfGFP-mediated fluorescence for each recombinant $E$. coli strain was measured (475/540 nm ex/em), and each data point shows the amount of fluorescence emitted by a single strain. The long horizontal bars show the mean of the replicate data, and error bars show \pm 1 SD $\left(n=6\right.$ or 12). $\left(^{*}\right) P$-value $<0.05,\left({ }^{* * *}\right) P$-value $<0.005,\left({ }^{* * * *}\right) P$-value $<0.0005$.

containing pTEXY::gltA-sfGFP and empty pREXY (no GcvB). Thus, GcvB-mediated repression of gltA expression was also abrogated by mutation of the $g c v B$ sRNA seed region. Finally, we tested the fluorescence of the E. coli strain containing both of the mutated plasmids, pTEXY:: glt $A_{M S R 1}-s f G F P$ and $\mathrm{pREXY}: \mathrm{gcv}_{\mathrm{MSR} 2}$, containing swapped seed regions but which are still complementary to each other. The strain containing these plasmids showed significantly reduced fluorescence compared to each of the strains containing the following plasmid pairs: pTEXY::gltA-sfGFP and empty pREXY, pTEXY::glt $A_{M S R 1}-s f G F P$ and pREXY::gcvB, and pTEXY::gltA-sfGFP and pREXY::gcvB $B_{M S R 2}$. Therefore, when the seed regions of both the GcvB sRNA and the mRNA target are mutated but in a complementary fashion, GcvB-mediated repression is restored, confirming that there is a direct interaction between the two predicted seed binding regions and that this level of binding is sufficient for the repression of $\mathrm{GcvB}$ expression.

\section{DISCUSSION}

In this study we have shown, using deep sequencing transcriptomic analyses and northern blotting, that the P. multocida GcvB is strongly expressed at early- and mid-exponential growth phases but displays highly reduced expression during late-exponential growth. These data correlate well with the known expression profile of GcvB in E. coli (Argaman et al. 2001) and S. Typhimurium (Sharma et al. 2007) and support the predicted function of $\mathrm{GcvB}$ as a repressor that acts primarily during growth under nutrient-rich conditions. We also examined the $5^{\prime}$ start of the GcvB sRNA expressed by the P. multocida strain VP161 using both primer extension and $5^{\prime}$ RACE. Primer extension identified the starting base as being positioned 1-2 bp downstream from the known start for the GcvB transcript in E. coli and $S$. Typhimurium. In contrast, experiments using $5^{\prime}$ RACE identified the transcript start was located 2 bp upstream of the start in E. coli and S. Typhimurium. The $5^{\prime}$ RACE method is considered the superior method for determining transcript starts as the $5^{\prime}$ end of the RNA is protected from degradation by the addition of an adapter. Therefore, we conclude the $P$. multocida GcvB begins with the sequence $5^{\prime}$-AUACUUAAU- $3^{\prime}$.

In order to identify the $P$. multocida GcvB regulon, we analyzed the proteome of the wild-type strain, a $g_{c} v B$ mutant, a $g c v B$ mutant containing empty vector and a GcvB overexpression strain. Nearly four times as many $P$. multocida proteins were identified as differentially produced in the $g c v B$ overexpression strain than in the GcvB-deficient strains. Quantitative qRTPCR showed that the level of GcvB in the overexpression strain was increased by approximately 70 -fold compared to the wild-type strain at early-exponential growth phase when the proteomics was performed. Therefore, we propose that the overexpression of GcvB to this level may lead to some off-target effects via nonspecific binding, as has been observed for other sRNAs (Storz et al. 2011).

The P. multocida $g c v B$ mutant displayed normal growth in rich medium, was unaffected by acid stress and showed no change in phenotype (compared to the parent strain) with respect to biofilm formation. This is in contrast to what has been reported for other species; $g c v B$ mutants constructed in E. coli, S. Typhimurium and $Y$. pestis all show a decreased growth rate in rich media, and inactivation of $g c v B$ in E. coli results in cells with decreased biofilm formation and decreased tolerance to acid stress (McArthur et al. 2006; Sharma et al. 2007; Jin et al. 2009; Mika and Hengge 2014). It is perhaps unsurprising that the $P$. multocida $g c v B$ mutant did not show a change in the ability to form a biofilm. Indeed, our data suggest that wild-type VP161 forms very poor single species biofilms. Moreover, with respect to acid tolerance, P. multocida is considered a bite wound and respiratory/ mucosal pathogen and, unlike enteric organisms, is unlikely to encounter strongly acidic conditions. However, the $P$. multocida $g c v B$ overexpression strain did show an increased lag-phase during growth when compared to wild-type VP161. It has been previously shown that during lag phase the glycolysis pathway is predominantly used to produce energy (Rolfe et al. 2012). An important enzyme in the Krebbs cycle is citrate synthase, which in $P$. multocida is encoded by the GcvB target, gltA. Therefore, the increase in lag phase displayed by 
the P. multocida $g c v B$ overexpression strain may in part be the result of decreased production of GltA due to increased GcvB binding to gltA transcripts. However, as this strain significantly overexpresses GcvB, it is acknowledged that the likely off-target effects on multiple other proteins may also play a role.

Of the proteins that showed either increased (36) or decreased (10) production in the GcvB-deficient P. multocida strains analyzed, 31 (27 increased, four decreased) were predicted to be involved in amino acid biosynthesis and transport, and pathway analyses indicated that GcvB specifically affects the biosynthesis of at least 14 different amino acids. Therefore, our data suggest that the P. multocida GcvB acts primarily to repress the production and transport of amino acids during the early growth stages, likely as a means to conserve energy when nutrients are abundant. In E. coli and $S$. Typhimurium the role of $\mathrm{GcvB}$ is also to repress amino acid biosynthesis and transport when nutrients are in plentiful supply. However, in these species GcvB shows a preponderance for regulation of amino acid transporters [ $>60 \%$ of GcvB targets (Sharma et al. 2011)]. In P. multocida this situation appears to be reversed, with the majority of the regulated proteins $(\sim 75 \%)$ being directly involved in the biosynthesis of amino acids. A comparison of the targets regulated by GcvB in P. multocida, E. coli, and S. Typhimurium identified four that were GcvB-regulated in all three species (GdhA, OppA, SerA, and DppA), two targets that were GcvB-regulated in both P. multocida and S. Typhimurium (PlpB and ThrA) and two targets that were GcvB-regulated in both P. multocida and E. coli (OppB and LysC). Thus, while the general function of GcvB as a controller of amino acid biosynthesis and transport has been conserved across the species, the precise GcvB targets show significant diversity.

The production of the histidine biosynthesis proteins HisA, HisB, HisC, HisF, HisG, and HisH_1 was strongly increased (fold change ranging from 1.6- to 2.3-fold) in P. multocida lacking a functional $g c v B$; five of these proteins are predicted to be encoded within a single operon. In other bacteria, histidine production is regulated by multiple mechanisms including repression of transcription initiation and attenuation (Kulis-Horn et al. 2014), but to our knowledge GcvB has not been previously linked with control of histidine biosynthesis. HisD encodes a histidinol dehydrogenase that has also been bioinformatically predicted to be a target of GcvB in the related Pasteurellaceae species A. pleuropneumoniae (Rossi et al. 2016). Moreover, specific attenuator sequences that target histidine production have been identified in A. pleuropneumoniae (Rossi et al. 2016).

Of the $27 \mathrm{GcvB}$ targets shown in Table 1, 71\% also showed increased production in a P. multocida strain VP161 hfq mutant (Mégroz et al. 2016). This indicates that the action of the P. multocida $\mathrm{GcvB}$ on many of the putative mRNA targets is dependent on the chaperone activity of Hfa, which mediates the docking of an sRNA onto its mRNA target. The reliance of $\mathrm{GcvB}$ on $\mathrm{Hfq}$ for binding to certain mRNA targets has also been demonstrated in E. coli (Pulvermacher et al. 2008). Compared to protein levels in the wild-type VP161, the predicted GcvB target SpeF showed increased production in the $g c v B$ mutant during early-exponential growth, when P. multocida GcvB has been shown to be most active. In contrast, SpeF showed decreased production in the P. multocida $h f q$ mutant during mid-exponential growth, indicating that other sRNAs may act upon SpeF at later growth phases.

Previously, it was proposed that during the late stages of $P$. multocida infection the in vivo environment is nutrient poor (Boyce et al. 2002). Under these conditions, we would predict that the levels of $g c v B$ gene expression would be low, thus allowing the expression of $g c v B$ mRNA targets involved in amino acid biosynthesis and transport. Supporting this prediction, four of the genes encoding putative GcvB mRNA targets, aspC, $d p p A$, $g d h A$, and gltA had increased expression (fold changes ranging from 1.8 to 11.3) during in vivo growth in chickens (Boyce et al. 2002). It is possible that GcvB plays an important role in the regulation of these targets in vivo. However, as yet we have no direct evidence of reduced GcvB expression during growth in vivo as the previous microarray experiments (Boyce et al. 2002) did not include DNA spots representing any sRNAs. In our current study, the glutamate dehydrogenase, GdhA, was identified as the most highly differentially produced protein (fivefold increase) following GcvB inactivation. GdhA catalyzes the conversion of L-glutamate to 2-oxoglutarate, releasing $\mathrm{NH}_{3}$ and $\mathrm{NADPH}$ which then allows for the production of all amino acids within the cell (Reitzer 1996). Interestingly, a P. multocida gdhA mutant belonging to the capsular type B and LPS serotype/genotype 2, was attenuated for virulence and was used as an effective vaccine against hemorrhagic septicemia in buffalo (Rafidah et al. 2012).

Comparative bioinformatic analysis using the gene sequences for 27 of the P. multocida GcvB mRNA targets, allowed for the identification of the predicted GcvB sRNA seed region (initial sRNA-mRNA binding site) consisting of 5'-AACACAACAT-3'. This sequence was highly conserved in a large number of the putative mRNA targets (Fig. 3C) and the complementary sequence of this seed region was present in the P. multocida GcvB sRNA. This seed binding sequence is $2 \mathrm{nt}$ longer than the characterized seed binding regions of the GcvB sRNA molecules encoded by E. coli and $S$. Typhimurium but importantly contains the same core region sequence, $5^{\prime}$-CACAACAT-3' (Urbanowski et al. 2000; McArthur et al. 2006; Pulvermacher et al. 2008; Sharma et al. 2011). The internal section of this binding region was confirmed as essential for GcvB interaction with the target gltA mRNA using the GFP translational reporter assay in E. coli, where substitution of these bases in either the mRNA target, gltA, or the sRNA, GcvB, decreased the interaction between the RNA molecules. Complementary substitution of bases in the sRNA and mRNA target allowed for restoration of binding efficiency and a concomitant decrease in GFP production. 
The predicted seed region within each of the negatively regulated P. multocida GcvB target mRNAs was mapped relative to the start codon of the gene. Similar to what has been observed in other bacteria (Bobrovskyy et al. 2015), most of the GcvB-specific seed regions mapped within the ribosome binding footprint, which we predict would allow GcvB to occlude the RBS and block translation of the target mRNA. However, some were located upstream of the ribosome binding footprint; this included the seed region sequence in thr $A$, which was located $\sim 43 \mathrm{nt}$ upstream of the ribosome binding footprint. The distal position of the seed region relative to the RBS has also been noted in some GcvB mRNA targets in E. coli and $S$. Typhimurium. In these instances it is thought that the CA-rich sequence within the seed region acts as a translational enhancer element and the binding of GcvB to this region blocks this enhanced translation (Sharma et al. 2007; Yang et al. 2014). Interestingly, 12 of the P. multocida mRNA targets (his B, metB, purC, msrA, metC_2, hisA, $p l p B, d p p A, \arg G, o p p A$, hisF, and $g d h A$ ) had the seed region sequence located on or after the start codon. Binding of an sRNA molecule soon after the translational start codon on the mRNA target is predicted to affect ribosome binding and translation of a gene because the ribosome footprint can extend from the -39 to the +19 nt (Hüttenhofer and Noller 1994; Sharma et al. 2007). Indeed, inhibitory interactions between the sRNA RybB and the mRNA target ompN in $S$. Typhimurium occur at +5 to $+20 \mathrm{nt}$ from the start codon (Bouvier et al. 2008).

The predicted seed region within the $g d h A$ mRNA (position +40$)$ is significantly downstream from the ribosome footprint region. Previous studies in $S$. Typhimurium have suggested that the sRNA-mRNA interactions between $g d h A$ and GcvB may include a second highly conserved GcvB binding site called R2 (Fig. 1). There is very limited evidence that R2 is definitively involved in any specific sRNA-mRNA binding interactions, as the study showed that deleting the $\mathrm{R} 2$ region of GcvB did not abrogate binding to the gdhA transcript (Sharma et al. 2011; Melamed et al. 2016). The R2 region is located downstream from the primary seed region and is present in GcvB from all bacterial species analyzed (Fig. 1; Sharma et al. 2011; Melamed et al. 2016). Future work will assess the importance of this $\mathrm{R} 2$ region in P. multocida.

This study has characterized the GcvB regulon in P. multocida strain VP161 and identified the seed binding regions required for interaction between GcvB and its targets. Many of the mRNA targets identified are required for the biosynthesis and transport of amino acids. Thus, the correct temporal expression of GcvB is likely to be important for growth of this pathogen in a nutrient depleted environment, such as in vivo during late-stage infection. While the GcvB target-binding site is well conserved between $P$. multocida and E. coli, and the GcvB-regulated genes in both species are primarily involved in amino acid biosynthesis and transport, the precise genes controlled by GcvB in the two species are quite different. These data are the first functional charac- terization of sRNA regulation in the Pasteurellaceae family; future studies will focus on identifying the role of GcvB and other sRNAs in vivo during P. multocida infections.

\section{MATERIALS AND METHODS}

\section{Bacterial strains, media, plasmids, and growth conditions}

All bacterial strains and plasmids used in this study are listed in Supplemental Table S2. P. multocida strains were routinely cultured in Heart Infusion (HI) broth (Oxoid). E. coli strains were routinely grown in Luria-Bertani (LB) broth (Oxoid). For solid media, 1.0\%$1.5 \%(\mathrm{w} / \mathrm{v})$ agar was added to the media. When required, media were supplemented with the appropriate antibiotics; kanamycin $(50 \mu \mathrm{g} / \mathrm{mL})$, spectinomycin $(50 \mu \mathrm{g} / \mathrm{mL})$, or ampicillin $(100 \mu \mathrm{g} / \mathrm{mL})$.

\section{DNA manipulations}

Genomic DNA was purified using the Genomic DNA Extraction Kit (RBC Bioscience Corp.), and plasmid DNA was extracted using the NucleoSpin Plasmid Kit (Macherey-Nagel GmbH \& Co. $\mathrm{KG}$ ), according to the manufacturer's instructions. For cloning experiments, restriction endonucleases (New England Biolabs) and ligase (Roche) were used according to the manufacturer's instructions. PCR amplifications were performed using Taq DNA Polymerase (Roche) or Phusion High Fidelity DNA Polymerase (Roche) with oligonucleotides (primers) manufactured by SigmaAldrich. The primers used in this study are listed in Supplemental Table S1. PCR products were purified using the NucleoSpin Gel and PCR Clean-up Kit (Macherey-Nagel). Sequencing reactions were performed with whole genomic DNA, plasmid DNA or PCR products as template as previously described (Harper et al. 2013). DNA sequences were analyzed using VectorNTI (Invitrogen), Clustal Omega (EBI), and BLAST (NCBI).

\section{Construction of a $P$. multocida gcv $B$ mutant}

To inactivate $g c v B$ in the P. multocida strain VP161, TargeTron mutagenesis (Sigma-Aldrich) was used as previously described (Steen et al. 2010) but with the following modifications. The group II intron within the E. coli-P. multocida TargeTron shuttle vector, pAL953 (Harper et al. 2013), was retargeted to $g c v B$ using the PCR amplification method described in the TargeTron manual. The primers BAP7565, BAP7566, and BAP7567 (Supplemental Table S1) were designed using the TargeTron design site (SigmaAldrich). The resulting plasmid, pAL1170 (Supplemental Table S2), was used to transform $P$. multocida strain VP161 by electroporation, and mutants containing a TargeTron group II intron insertion in $g c v B$ were identified as previously described (Harper et al. 2013).

\section{Construction of a P. multocida GcvB overexpression strain}

The $g c v B$ gene from $P$. multocida VP161 was amplified using BAP7585 and BAP7586 (Supplemental Table S1), digested with 
BamHI and SalI, and cloned into similarly digested pPBA1100s. The resulting plasmid, pAL1190, and the empty vector PPBA1100s were separately used to transform the P. multocida gcvB mutant AL2677 via electroporation, producing strains AL2864 and AL2862, respectively (Supplemental Table S2).

\section{Heterologous expression of the $E$. coli hfq gene in $P$. multocida}

The $h f q$ gene from E. coli DH5a was amplified using BAP7850 and BAP7851 (Supplemental Table S1), digested with BamHI and SalI then cloned into the P. multocida expression plasmid, pAL99T. The resulting plasmid, pAL1266, was used to transform the P. multocida hfq mutant AL2521 (Mégroz et al. 2016), producing the strain AL2838.

\section{Hyaluronic acid capsule assay}

P. multocida strains were grown in HI broth (in biological triplicate) supplemented with the appropriate antibiotics (where required) to mid-exponential growth phase $\left(\mathrm{OD}_{600}=0.6\right)$. Capsule was extracted from washed cells and the amount of capsular material measured using a hyaluronic acid assay as described previously (Chung et al. 2001).

\section{Response to acid stress}

Acidic HI broth was prepared by addition of $37 \%$ (v/v) hydrochloric acid $(\mathrm{HCl})$ to $\mathrm{HI}$ until $\mathrm{pH} 4.6$ was reached. Triplicate overnight cultures were prepared for each $P$. multocida strain and supplemented with Kanamycin where required to maintain the plasmid. Each culture was diluted 1:100 in fresh HI broth and grown until early exponential phase $\left(\mathrm{OD}_{600}=0.2\right)$. A $1 \mathrm{~mL}$ aliquot of this early exponential phase $\left(\mathrm{OD}_{600}=0.2\right)$ culture was then added to $3 \mathrm{~mL}$ of acidified $\mathrm{HI}$ broth, without antibiotics, and incubated at $37^{\circ} \mathrm{C}$ for $15 \mathrm{~min}$ with shaking. Following incubation, $12 \mathrm{~mL}$ of basic $\mathrm{HI}$ broth was added to neutralize the culture. Appropriate dilutions of each culture were plated onto $\mathrm{HI}$ agar in duplicate and after $16 \mathrm{~h}$ incubation colonies were enumerated.

\section{Biofilm formation assay}

Cultures representing each bacterial strain were grown to mid-exponential growth phase $\left(\mathrm{OD}_{600}=0.6\right)$, then $100 \mu \mathrm{L}$ of the diluted culture (1:100) was added to four wells of a sterile 96-well plate which was then incubated overnight at $37^{\circ} \mathrm{C}$ without shaking to allow for biofilm formation. Following incubation, the plate was washed three times with $\mathrm{dH}_{2} \mathrm{O}$ to remove planktonic bacteria. Remaining bacteria were stained with $125 \mu \mathrm{L}$ of $0.1 \%(\mathrm{w} / \mathrm{v})$ crystal violet and incubated for $10 \mathrm{~min}$ at room temperature. Excess stain was removed by washing with $\mathrm{dH}_{2} \mathrm{O}$ three times. To resolubilize the crystal violet, $200 \mu \mathrm{L}$ of $95 \%$ (v/v) EtOH was added to each well, incubated for $15 \mathrm{~min}$ and then mixed well. A $125 \mu \mathrm{L}$ aliquot of each well was transferred to a well of an optically clear flat bottomed 96-well plate and the optical density determined using a Tecan Infinite M200 plate reader.

\section{RNA extraction, qRT-PCR, and whole-genome transcriptomic analyses by RNA-seq}

P. multocida RNA extractions were performed as described previously (Boyce et al. 2002) but with the following modifications. Duplicate bacterial cultures were grown in $\mathrm{HI}$ broth to $\mathrm{OD}_{600}=$ 0.2 (early-exponential growth phase), $\mathrm{OD}_{600}=0.6$ (mid-exponential growth phase), or $\mathrm{OD}_{600}=1.0$ (late-exponential growth phase). Killing buffer was omitted from the RNA extraction method. Following DNase treatment of the samples, RNA was further purified by phenol:choloform extraction using 5Prime phase lock gel tubes as per the manufacturer's instructions (Quanta Biosciences). RNA-seq library preparation, sequencing on an Illumina HiSeq, and mapping and differential expression analysis were carried out as previously described (Mégroz et al. 2016). For the RNA-seq analyses, the average number of reads mapped across samples was 5647690.83 , and of these an average of $99.7 \%$ mapped to the P. multocida VP161 genome, giving an average read depth of 2701.76 reads per gene. qRT-PCR was performed using the AffinityScript cDNA Synthesis Kit (Agilent) and Brilliant II SYBR Green qPCR Kit (Agilent) as per the manufacturer's instructions using the Eppendorf Realplex Mastercycler. Reverse transcription reactions, both plus and minus reverse transcriptase (+RT and $-\mathrm{RT}$, respectively), were performed in biological triplicate, with each $+\mathrm{RT}$ reaction being measured in technical triplicate and each $-\mathrm{RT}$ reaction being measured in technical duplicate. Data were analyzed to ensure melt curves identified that only a single product was formed in each reaction and - RT controls did not amplify any products within 10 cycles of the experimental reactions.

\section{Northern blotting}

Northern blotting analysis was performed using the DIG Northern Starter Kit version 10 (Roche) as per the manufacturer's instructions, with the following modifications. A total of $8 \mu \mathrm{g}$ of RNA was separated by agarose/formaldehyde gel electrophoresis and the separated products transferred to a nylon membrane by capillary electrophoresis. A GcvB-specific probe was amplified from P. multocida VP161 genomic DNA using BAP7888 and BAP7957; BAP7957 contains a T7 RNA polymerase promoter sequence at the $5^{\prime}$ end. The PCR product was then used in an in vitro transcription reaction using T7 RNA polymerase and 10X DIG labeled RNA mix (Promega).

\section{Proteomics analysis}

Total proteomes of the wild-type $P$. multocida VP161 and the $g c v B$ mutant (in triplicate), were determined using nano-liquid chromatography coupled with tandem MS, following isotopic labeling with heavy and light formaldehyde as described previously (Mégroz et al. 2016).

Total proteomes of the wild-type P. multocida VP161, the $g c v B$ mutant with empty vector and the GcvB overexpression strain were determined using label-free quantitative proteomics. Cells were grown in biological triplicate in $\mathrm{HI}$ broth to early-exponential growth phase $\left(\mathrm{OD}_{600}=0.2\right)$ and pelleted by centrifugation. Cell pellets were lysed in $1 \% \mathrm{w} / \mathrm{v}$ SDC (sodium deoxycholate; SigmaAldrich), $100 \mathrm{mM}$ Tris $(\mathrm{pH}=8.1)$ and further homogenized on a Soniprep 150 Plus Sonicator (MSE). The protein concentration 
was determined using a BCA Assay Kit (Pierce). A $200 \mu \mathrm{g}$ aliquot of each total protein sample was denatured using $10 \mathrm{mM}$ TCEP (Thermo Scientific), and free cysteine residues were alkylated with $40 \mathrm{mM}$ chloroacetamide (Sigma-Aldrich). Trypsin Gold (Promega) was used to digest the proteins and SDC removed by extraction with water-saturated ethyl acetate. All samples were desalted using P-10 ZipTip columns (Agilent, OMIX-Mini Bed 96 $\mathrm{C} 18)$, vacuum-dried and reconstituted in buffer A $(0.1 \%$ formic acid, $2 \%$ acetonitrile) prior to mass spectrometry.

Using a Dionex UltiMate 3000 RSLCnano system equipped with a Dionex UltiMate 3000 RS autosampler, the samples were loaded via an Acclaim PepMap 100 trap column $(100 \mu \mathrm{m} \times 2 \mathrm{~cm}$, nanoViper, C18, $5 \mu \mathrm{m}, 100 \AA$; Thermo Scientific) onto an Acclaim PepMap RSLC analytical column $(75 \mu \mathrm{m} \times 50 \mathrm{~cm}$, nanoViper, C18, $2 \mu \mathrm{m}$, $100 \AA$; Thermo Scientific). The peptides were separated using increasing concentrations of buffer B ( $80 \%$ acetonitrile/ $0.1 \%$ formic acid) for $158 \mathrm{~min}$ and analyzed with a QExactive Plus mass spectrometer (Thermo Scientific) operated in data-dependent acquisition mode using in-house, LFQ-optimized parameters.

Acquired .raw files were analyzed with MaxQuant (Cox and Mann 2008) to globally identify and quantify proteins across the various conditions. Statistical analyses for identification of differentially produced proteins were performed using the Limma package within $\mathrm{R}$ studio, where FDR is derived from the BenjaminiHochberg procedure. Differentially produced proteins were identified as proteins with $a \geq 0.59 \log _{2}$ fold change and an FDR $\leq 0.05$. The proteomics data have been deposited in ProteomeXchange via the PRIDE database with identifier PXD007719.

\section{Fluorescent primer extension}

Fluorescent primer extension was performed as described previously (Lloyd et al. 2005; Steen et al. 2010) with the following modifications. RNA was isolated from P. multocida $\mathrm{VP} 161$ at $\mathrm{OD}_{600}=0.2$. For cDNA synthesis, $10 \mu \mathrm{g}$ of total RNA was used as template with the 6-carboxy fluorescein amidite (6-FAM) labeled primer, BAP7962 or BAP8190 (Supplemental Table S1). Dried samples were analyzed using an ABI 3730xl DNA Analyzer (Thermo Fisher Scientific) located at the Australian Genome Research Facility (AGRF, Melbourne).

\section{5' RACE}

5' RACE was performed with $10 \mu \mathrm{g}$ of RNA isolated from P. multocida VP161 using the Firstchoice RLM-RACE Kit (Applied Biosystems) according to the manufacturer's instructions with the following modifications. The reverse transcription step was replaced with the cDNA synthesis protocol used for fluorescent primer extension (above) using the nonfluorescent GcvB-specific primer BAP7889. The cDNA generated was resuspended in $30 \mu \mathrm{L}$ of nuclease-free water; $1 \mu \mathrm{L}$ was used in the first, nested PCR using the primer BAP7889 together with the commercially supplied $5^{\prime}$ RACE outer primer (Applied Biosystems). PCR reaction conditions were as follows; $94^{\circ} \mathrm{C}$ for $3 \mathrm{~min}$, followed by 35 cycles consisting of $94^{\circ} \mathrm{C} 30 \mathrm{sec}, 62^{\circ} \mathrm{C} 30 \mathrm{sec}, 72^{\circ} \mathrm{C} 1 \mathrm{~min}$, followed by a final extension step of $72^{\circ} \mathrm{C}$ for $7 \mathrm{~min}$. The PCR product was then purified and $1 \mu \mathrm{L}$ was used in the second, nested PCR using the primer BAP7754 and the commercially supplied 5' RACE inner primer (Applied Biosystems) with the same PCR reaction conditions as described above. The nested PCR products generated were cloned into the vector pCR2.1 using the TOPO TA Cloning Kit (Thermo Fisher Scientific) according to the manufacturer's instructions. The nucleotide sequences of the cloned inserts were then determined using the vector-specific primer BAP612 in Sanger sequencing reactions.

\section{Coimmunoprecipitation of $\mathrm{GcvB}$ by $\mathrm{Hfq}$}

To test whether P. multocida GcvB bound Hfq, we used coimmunoprecipitation of total bacterial RNA by a FLAG-tagged Hfq, followed by high-throughput sequencing of the precipitated RNAs. Total RNA was prepared from P. multocida expressing a chromosomally encoded, C-terminal 3xFLAG-tagged $\mathrm{Hfq}$ and as a control also from the wild-type $P$. multocida expressing native Hfq. FLAG-tagged $\mathrm{Hfq}$, and any bound RNAs, were precipitated (three independent coimmunoprecipitation reactions) using anti-FLAG conjugated magnetic beads as previously described (Bilusic et al. 2014). RNAseq library preparation, sequencing on a NextSeq (Illumina), and mapping and differential expression analysis was carried out as previously described (Mégroz et al. 2016).

\section{Construction of plasmids for the two-plasmid GFP reporter assays}

To analyze $P$. multocida GcvB/gltA mRNA target interactions, a twoplasmid GFP reporter system was developed based on the previously described system of Urban and Vogel (2007). This system required the construction of two expression vectors, pTEXY, required for the expression of the $5^{\prime}$ end of the mRNA target, containing the GcvB seed/binding region fused to a gene encoding sfGFP (Corcoran et al. 2012), and pREXY, required for the expression of the sRNA molecule, GcvB. To generate pTEXY the unique BsgI site present in the E. coli plasmid pBR322 was first changed to a BssHII site using site-directed PCR mutagenesis to allow for future experiments that required this restriction site to be uniquely located in the cloned mRNA DNA fragments. Two PCR products representing the pBR322 nucleotides 1 to 1656 (position of BsgI site) and nucleotides 1653-4358 were amplified by PCR. The first PCR reaction amplified the pBR322 nucleotides 1-1656 using BAP7721, which anneals to the EcoRI region and BAP7720, which anneals to the BsgI region but contains an altered sequence to incorporate a BssHII site instead of BsgI. The second PCR reaction amplified the pBR322 nucleotides 1653-4358 using BAP7722, which anneals to the EcoRI region and BAP7719, which anneals to the BsgI region but contains a BssHII site instead of BsgI. The PCR products were digested with EcoRI and BssHII, ligated, and the mixture used to transform competent E. coli DH5a, to generate the plasmid, pAL1240 (Supplemental Table S2). To construct pTEXY, a pMAT plasmid containing a commercially synthesized DNA fragment (Life Technologies) encoding the $s f G F P$ gene (flanked by a HindIII and EcoRI restriction sites and under the control of the anhydrotetracycline (Atc)-inducible promoter, $\left.P_{\text {LtetO-1 }}\right)$, was digested with HindIII and EcoRI. The DNA fragment containing sfGFP was then gel-purified and ligated to HindIII and EcoRI-digested pAL1240 to generate the plasmid pTEXY (Supplemental Table S2).

The final expression plasmid containing the gltA-sfGFP fusion (pAL1257) was constructed by generating an XbaI/BglII-digested PCR fragment, using the primers BAP7747 and BAP7748, that represented the region -38 to +60 (relative to start codon) of the 
P. multocida gltA. The PCR fragment was digested, purified then ligated into the XbaI and BglII-digested pTEXY (Supplemental Table S2), such that expression would be under the control of the $P_{\text {LtetO-1 }}$ promoter. The pTEXY plasmid, pAL1290, containing gltA with a mutated seed region $\left(g l t A_{M S R}-s f G F P\right)$ was constructed in a similar manner with the exception that the forward primer BAP7964, containing the altered seed region sequence, was used for the amplification of the gltA-specific DNA (Supplemental Table S1).

To generate the base plasmid, pPBA1100S, used for the construction of sRNA expression plasmid pREXY, the DNA region (240 bp) encoding the $\mathrm{P}_{t p i}$ promoter was removed from the pAL99S vector (Harper et al. 2013) using EcoRI digestion followed by religation of the vector. This region was then replaced with a shorter DNA fragment $\left(96 \mathrm{bp}\right.$ ) containing the $\mathrm{P}_{t p i}$ promoter, amplified from P. multocida VP161 genomic DNA using BAP7638 and BAP7639 (containing HindIII and BamHI restriction sites, respectively), to ensure that transcription could begin as close as possible to the native sRNA (GcvB) start site. The BamHI/HindIII-digested PCR product was ligated to similarly digested pPBA1100S and the ligation mix used to transform E. coli DH5a. Recombinant colonies were selected on $\mathrm{HI}$ agar containing $50 \mu \mathrm{g} / \mu \mathrm{L}$ spectinomycin. Correct recombinant plasmids were confirmed by restriction analysis and DNA sequencing and one plasmid with the correct sequence designated pREXY (Supplemental Table S2).

The GcvB expression plasmid, pAL1197 (Supplemental Table S2), was constructed as follows. The region of the P. multocida VP161 genome encoding the putative $g c v B$ was amplified from VP161 genomic DNA using the primers BAP7632 and BAP7633 (both containing an XmaI site). The purified, XmaI-digested, PCR product was then ligated to XmaI-digested pREXY. The authenticity of the pAL1197 plasmid containing $g c v B$, was confirmed by PCR and DNA sequencing. The pREXY plasmid containing the mutated $g c v B_{M S R 2}$ (pAL1277, Supplemental Table S2) was constructed using splice overlap extension (SOE) PCR. Two PCR reactions were performed as follows. The reverse primer, BAP7951 and the forward primer BAP7950 (Supplemental Table S1), that overlap and anneal to the $g c v B$ gene region containing the predicted seed region, were paired with BAP7632 (forward primer located upstream of $g_{c} v B$ ) and BAP7633 (reverse primer located downstream from $g c v B$ ), respectively. Primers BAP7951 and BAP7950 contained sequence that changed the $g c v B$ seed region from $5^{\prime}$-GTTGTGT- $3^{\prime}$ to $5^{\prime}$ CAACACA $-3^{\prime}$. The two PCR fragments, representing the $5^{\prime}$ and $3^{\prime}$ ends of $g c v B$, were combined using a second PCR amplification with primers BAP7632 and BAP7633 (Supplemental Table S1) to produce the $g c v B_{M S R 2}$ fragment. The PCR product was then purified, digested with XmaI, and ligated to XmaI-digested pREXY.

\section{Whole-cell fluorescent measurements}

E. coli DH5a strains containing both a pTEXY-based plasmid (5' mRNA-sfGFP fusion expression) and a pREXY-based plasmid (sRNA expression) were grown on LB agar supplemented with $50 \mu \mathrm{g} / \mu \mathrm{L}$ ampicillin and $50 \mu \mathrm{g} / \mu \mathrm{L}$ spectinomycin. Cells representing each strain were harvested from each plate (biological triplicate), resuspended in $1 \mathrm{~mL}$ of $1 \times \mathrm{PBS}$ buffer, and the volume adjusted to give a final $\mathrm{OD}_{600}$ of 2.0. A $200 \mu \mathrm{L}$ aliquot of each cell suspension was added to a black flat bottomed 96-well microtiter plate (in triplicate). Fluorescence was measured using the Tecan Infinite M200 plate reader with an excitation/emission wavelength of 475/540 nm.

\section{Bioinformatic analysis}

Comparison of nucleotide and protein sequences was performed using BLAST (Camacho et al. 2009). The Rfam database version 12.2 (Burge et al. 2013) was used to compare the P. multocida $g c v B$ sequence to known RNAs. The MEME motif identification website with MEME motif finder version 4.11.2 (Bailey et al. 2009) was used to identify potential GcvB binding sites in putative mRNA targets and these were then further analyzed using Clustal Omega (Sievers et al. 2011). Sequence data were analyzed and recombinant DNA molecules were designed using VectorNTI version 11 (Invitrogen). GcvB-regulated genes were mapped to the appropriate metabolic pathways using SmartTables (Travers et al. 2013) and pathway overview (Paley and Karp 2006; Karp et al. 2010) within the Biocyc database collection website (Caspi et al. 2016). Metabolic pathways were visualized using flow charts obtained from the Kyoto Encyclopedia of Genes and Genomes (KEGG) database (Kanehisa et al. 2016). The Mfold webserver was used with default parameters to determine RNA secondary structures (Zuker 2003).

\section{SUPPLEMENTAL MATERIAL}

Supplemental material is available for this article.

\section{ACKNOWLEDGMENTS}

This work was supported in part by the Australian Research Council discovery project grant number 150103715 . E.G. is supported by a Monash University MBio Postgraduate Discovery Scholarship.

Received August 2, 2017; accepted February 1, 2018.

\section{REFERENCES}

Argaman L, Hershberg R, Vogel J, Bejerano G, Wagner EG, Margalit H, Altuvia S. 2001. Novel small RNA-encoding genes in the intergenic regions of Escherichia coli. Curr Biol 11: 941-950.

Baddal B, Muzzi A, Censini S, Calogero RA, Torricelli G, Guidotti S, Taddei AR, Covacci A, Pizza M, Rappuoli R, et al. 2015. Dual RNA-seq of nontypeable haemophilus influenzae and host cell transcriptomes reveals novel insights into host-pathogen cross talk. MBio 6: e01765-15.

Bailey TL, Boden M, Buske FA, Frith M, Grant CE, Clementi L, Ren J, Li WW, Noble WS. 2009. MEME SUITE: tools for motif discovery and searching. Nucleic Acids Res 37: W202-W208.

Bilusic I, Popitsch N, Rescheneder P, Schroeder R, Lybecker M. 2014. Revisiting the coding potential of the E. coli genome through Hfa co-immunoprecipitation. RNA Biol 11: 641-654.

Bobrovskyy M, Vanderpool CK, Richards GR. 2015. Small RNAs regulate primary and secondary metabolism in Gram-negative bacteria. Microbiol Spectr 3. doi: 10.1128/microbiolspec.MBP-0009-2014.

Bosch M, Garrido E, Llagostera M, Pérez de Rozas AM, Badiola I, Barbé J. 2002. Pasteurella multocida exbB, exbD and tonB genes are physically linked but independently transcribed. FEMS Microbiol Lett 210: 201-208.

Bouvier M, Sharma CM, Mika F, Nierhaus KH, Vogel J. 2008. Small RNA binding to $5^{\prime}$ mRNA coding region inhibits translational initiation. Mol Cell 32: 827-837.

Boyce JD, Adler B. 2006. How does Pasteurella multocida respond to the host environment? Curr Opin Microbiol 9: 117-122.

Boyce JD, Wilkie I, Harper M, Paustian ML, Kapur V, Adler B. 2002. Genomic scale analysis of Pasteurella multocida gene expression 
during growth within the natural chicken host. Infect Immun 70: 6871-6879.

Boyce JD, Seemann T, Adler B, Harper M. 2012. Pathogenomics of Pasteurella multocida. Curr Top Microbiol Immunol 361: 23-38.

Burge SW, Daub J, Eberhardt R, Tate J, Barquist L, Nawrocki EP, Eddy SR, Gardner PP, Bateman A. 2013. Rfam 11.0: 10 years of RNA families. Nucleic Acids Res 41: D226-D232.

Camacho C, Coulouris G, Avagyan V, Ma N, Papadopoulos J, Bealer K, Madden TL. 2009. BLAST+: architecture and applications. BMC Bioinformatics 10: 421.

Caspi R, Billington R, Ferrer L, Foerster H, Fulcher CA, Keseler IM, Kothari A, Krummenacker M, Latendresse M, Mueller LA, et al. 2016. The MetaCyc database of metabolic pathways and enzymes and the BioCyc collection of pathway/genome databases. Nucleic Acids Res 44: D471-D480.

Chung JY, Wilkie I, Boyce JD, Townsend KM, Frost AJ, Ghoddusi M, Adler B. 2001. Role of capsule in the pathogenesis of fowl cholera caused by Pasteurella multocida serogroup A. Infect Immun 69: 2487-2492.

Coornaert A, Chiaruttini C, Springer M, Guillier M. 2013. Posttranscriptional control of the Escherichia coli PhoQ-PhoP twocomponent system by multiple sRNAs involves a novel pairing region of GcvB. PLoS Genet 9: e1003156.

Corcoran CP, Podkaminski D, Papenfort K, Urban JH, Hinton JC, Vogel J. 2012. Superfolder GFP reporters validate diverse new mRNA targets of the classic porin regulator, MicF RNA. Mol Microbiol 84: 428-445.

Cox J, Mann M. 2008. MaxQuant enables high peptide identification rates, individualized p.p.b.-range mass accuracies and proteomewide protein quantification. Nat Biotechnol 26: 1367-1372.

Desnoyers G, Bouchard MP, Massé E. 2013. New insights into small RNA-dependent translational regulation in prokaryotes. Trends Genet 29: 92-98.

Fuller TE, Kennedy MJ, Lowery DE. 2000. Identification of Pasteurella multocida virulence genes in a septicemic mouse model using signature-tagged mutagenesis. Microb Pathog 29: 25-38.

Gottesman S, Storz G. 2011. Bacterial small RNA regulators: versatile roles and rapidly evolving variations. Cold Spring Harb Perspect Biol 3: a003798.

Harper M, Cox AD, St Michael F, Wilkie IW, Boyce JD, Adler B. 2004. A heptosyltransferase mutant of Pasteurella multocida produces a truncated lipopolysaccharide structure and is attenuated in virulence. Infect Immun 72: 3436-3443.

Harper M, St Michael F, John M, Vinogradov E, Steen JA, van Dorsten L, Steen JA, Turni C, Blackall PJ, Adler B, et al. 2013. Pasteurella multocida Heddleston serovar 3 and 4 strains share a common lipopolysaccharide biosynthesis locus but display both inter- and intrastrain lipopolysaccharide heterogeneity. J Bacteriol 195: 4854-4864.

Hüttenhofer A, Noller HF. 1994. Footprinting mRNA-ribosome complexes with chemical probes. EMBO J 13: 3892-3901.

Jin Y, Watt RM, Danchin A, Huang JD. 2009. Small noncoding RNA GcvB is a novel regulator of acid resistance in Escherichia coli. BMC Genomics 10: 165.

Kanehisa M, Sato Y, Kawashima M, Furumichi M, Tanabe M. 2016. KEGG as a reference resource for gene and protein annotation. Nucleic Acids Res 44: D457-D462.

Karp PD, Paley SM, Krummenacker M, Latendresse M, Dale JM, Lee TJ, Kaipa P, Gilham F, Spaulding A, Popescu L, et al. 2010. Pathway Tools version 13.0: integrated software for pathway/genome informatics and systems biology. Brief Bioinform 11: 40-79.

Kulis-Horn RK, Persicke M, Kalinowski J. 2014. Histidine biosynthesis, its regulation and biotechnological application in Corynebacterium glutamicum. Microb Biotechnol 7: 5-25.

Lloyd AL, Marshall BJ, Mee BJ. 2005. Identifying cloned Helicobacter pylori promoters by primer extension using a FAM-labelled primer and GeneScan analysis. J Microbiol Methods 60: 291-298.

McArthur SD, Pulvermacher SC, Stauffer GV. 2006. The Yersinia pestis $g c v B$ gene encodes two small regulatory RNA molecules. $B M C$ Microbiol 6: 52 .
Mégroz M, Kleifeld O, Wright A, Powell D, Harrison P, Adler B, Harper M, Boyce JD. 2016. The RNA-binding chaperone Hfq is an important global regulator of gene expression in Pasteurella multocida and plays a crucial role in production of a number of virulence factors, including hyaluronic acid capsule. Infect Immun 84: 1361-1370.

Melamed S, Peer A, Faigenbaum-Romm R, Gatt YE, Reiss N, Bar A, Altuvia Y, Argaman L, Margalit H. 2016. Global mapping of small RNA-target interactions in bacteria. Mol Cell 63: 884-897.

Mika F, Hengge R. 2014. Small RNAs in the control of RpoS, CsgD, and biofilm architecture of Escherichia coli. RNA Biol 11: 494-507.

Paley SM, Karp PD. 2006. The Pathway Tools cellular overview diagram and Omics Viewer. Nucleic Acids Res 34: 3771-3778.

Pulvermacher SC, Stauffer LT, Stauffer GV. 2008. The role of the small regulatory RNA GcvB in $\mathrm{GcvB} / \mathrm{mRNA}$ posttranscriptional regulation of $о p p A$ and $A p p A$ in Escherichia coli. FEMS Microbiol Lett 281: $42-50$.

Rafidah O, Zamri-Saad M, Shahirudin S, Nasip E. 2012. Efficacy of intranasal vaccination of field buffaloes against haemorrhagic septicaemia with a live $g d h A$ derivative Pasteurella multocida B:2. Vet Rec 171: 175.

Reitzer LJ. 1996. Ammonia assimilation and the biosynthesis of glutamine, glutamate, aspartate, asparagine, 1-alanine, and d-alanine. In Escherichia coli and Salmonella: cellular and molecular biology (ed. Neidhardt FC, et al.), Vol. 1, pp. 391-407. ASM Press, Washington, DC.

Rolfe MD, Rice CJ, Lucchini S, Pin C, Thompson A, Cameron AD, Alston M, Stringer MF, Betts RP, Baranyi J, et al. 2012. Lag phase is a distinct growth phase that prepares bacteria for exponential growth and involves transient metal accumulation. J Bacteriol 194: 686-701.

Rossi CC, Bossé JT, Li Y, Witney AA, Gould KA, Langford PR, Bazzolli DM. 2016. A computational strategy for the search of regulatory small RNAs in Actinobacillus pleuropneumoniae. RNA 22: 1373-1385.

Sharma CM, Darfeuille F, Plantinga TH, Vogel J. 2007. A small RNA regulates multiple $\mathrm{ABC}$ transporter mRNAs by targeting C/A-rich elements inside and upstream of ribosome-binding sites. Genes Dev 21: 2804-2817.

Sharma CM, Papenfort K, Pernitzsch SR, Mollenkopf HJ, Hinton JC, Vogel J. 2011. Pervasive post-transcriptional control of genes involved in amino acid metabolism by the Hfq-dependent GcvB small RNA. Mol Microbiol 81: 1144-1165.

Sievers F, Wilm A, Dineen D, Gibson TJ, Karplus K, Li W, Lopez R, McWilliam H, Remmert M, Soding J, et al. 2011. Fast, scalable generation of high-quality protein multiple sequence alignments using Clustal Omega. Mol Syst Biol 7: 539.

Steen JA, Steen JA, Harrison P, Seemann T, Wilkie I, Harper M, Adler B, Boyce JD. 2010. Fis is essential for capsule production in Pasteurella multocida and regulates expression of other important virulence factors. PLoS Pathog 6: e1000750.

Storz G, Vogel J, Wassarman KM. 2011. Regulation by small RNAs in bacteria: expanding frontiers. Mol Cell 43: 880-891.

Travers M, Paley SM, Shrager J, Holland TA, Karp PD. 2013. Groups: knowledge spreadsheets for symbolic biocomputing. Database (Oxford) 2013: bat061.

Urban JH, Vogel J. 2007. Translational control and target recognition by Escherichia coli small RNAs in vivo. Nucleic Acids Res 35: 1018-1037.

Urbanowski ML, Stauffer LT, Stauffer GV. 2000. The $g c v B$ gene encodes a small untranslated RNA involved in expression of the dipeptide and oligopeptide transport systems in Escherichia coli. Mol Microbiol 37: 856-868.

Wilkie IW, Harper M, Boyce JD, Adler B. 2012. Pasteurella multocida: diseases and pathogenesis. Curr Top Microbiol Immunol 361: 1-22.

Yang Q, Figueroa-Bossi N, Bossi L. 2014. Translation enhancing ACA motifs and their silencing by a bacterial small regulatory RNA. PLoS Genet 10: e1004026.

Zuker M. 2003. Mfold web server for nucleic acid folding and hybridization prediction. Nucleic Acids Res 31: 3406-3415. 

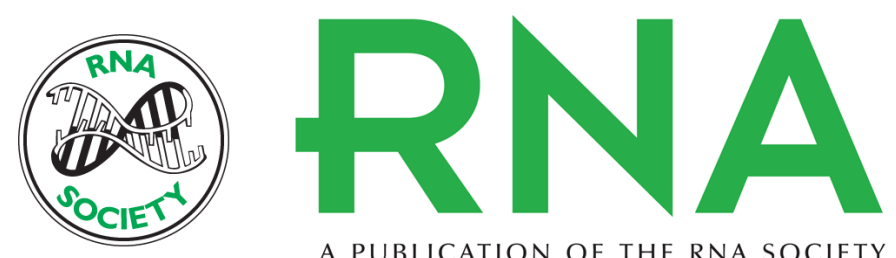

A PUBLICATION OF THE RNA SOCIETY

\section{Determination of the small RNA GcvB regulon in the Gram-negative bacterial pathogen Pasteurella multocida and identification of the GcvB seed binding region}

Emily L. Gulliver, Amy Wright, Deanna Deveson Lucas, et al.

RNA 2018 24: 704-720 originally published online February 12, 2018

Access the most recent version at doi:10.1261/rna.063248.117

Supplemental Material

References

Creative Commons License

Email Alerting Service
http://rnajournal.cshlp.org/content/suppl/2018/02/12/rna.063248.117.DC1

This article cites 49 articles, 11 of which can be accessed free at: http://rnajournal.cshlp.org/content/24/5/704.full.html\#ref-list-1

This article is distributed exclusively by the RNA Society for the first 12 months after the full-issue publication date (see http://rnajournal.cshlp.org/site/misc/terms.xhtml). After 12 months, it is available under a Creative Commons License (Attribution-NonCommercial 4.0 International), as described at http://creativecommons.org/licenses/by-nc/4.0/.

Receive free email alerts when new articles cite this article - sign up in the box at the top right corner of the article or click here.

To subscribe to $R N A$ go to:

http://rnajournal.cshlp.org/subscriptions 